\title{
JPEG Compression History Estimation for Color Images
}

\author{
Ramesh (Neelsh) Neelamani, Member, IEEE, Ricardo de Queiroz, Senior Member, IEEE, \\ Zhigang Fan, Senior Member, IEEE, Sanjeeb Dash, and Richard G. Baraniuk, Fellow, IEEE
}

\begin{abstract}
We routinely encounter digital color images that were previously compressed using the Joint Photographic Experts Group (JPEG) standard. En route to the image's current representation, the previous JPEG compression's various settings-termed its JPEG compression history $(\mathrm{CH})$-are often discarded after the JPEG decompression step. Given a JPEG-decompressed color image, this paper aims to estimate its lost JPEG CH. We observe that the previous JPEG compression's quantization step introduces a lattice structure in the discrete cosine transform (DCT) domain. This paper proposes two approaches that exploit this structure to solve the JPEG Compression History Estimation (CHEst) problem. First, we design a statistical dictionary-based CHEst algorithm that tests the various $\mathrm{CHs}$ in a dictionary and selects the maximum a posteriori estimate. Second, for cases where the DCT coefficients closely conform to a 3-D parallelepiped lattice, we design a blind lattice-based CHEst algorithm. The blind algorithm exploits the fact that the JPEG CH is encoded in the nearly orthogonal bases for the 3-D lattice and employs novel lattice algorithms and recent results on nearly orthogonal lattice bases to estimate the $\mathrm{CH}$. Both algorithms provide robust JPEG CHEst performance in practice. Simulations demonstrate that JPEG CHEst can be useful in JPEG recompression; the estimated CH allows us to recompress a JPEG-decompressed image with minimal distortion (large signal-to-noise-ratio) and simultaneously achieve a small file-size.
\end{abstract}

Index Terms-Color, compression, history, Joint Photographic Experts Group (JPEG), lattice, quantization, recompression.

\section{INTRODUCTION}

A DIGITAL color image is a collection of pixels with each pixel a three-dimensional (3-D) color vector. The vector elements specify the pixel's color with respect to a chosen color space; for example, $R G B, Y C b C r$, etc. [1], [2]. The Joint Photographic Experts Group (JPEG) is a commonly used standard to compress digital color images [3]. JPEG

Manuscript received June 12, 2004; revised April 29, 2005. This work was supported by grants from the National Science Foundation, Air Force Office of Scientific Research, Office of Naval Research, Defense Advanced Research Projects Agency, and the Texas Instruments Leadership University Program. The associate editor coordinating the review of this manuscript and approving it for publication was Dr. Giovanni Poggi.

R. Neelamani is with ExxonMobil Upstream Research Company, Houston, TX 77027-6019 USA (e-mail: neelsh@ rice.edu).

R. de Queiroz is with the Department of Electrical Engineering, Universidade de Brasilia, Brasilia, DF 70910-900, Brazil (e-mail: queiroz@ieee.org).

Z. Fan is with Xerox Research and Technology, Xerox Corporation, Webster, NY 14580 USA (e-mail: ZFan@ crt.xerox.com).

S. Dash is with the IBM T. J. Watson Research Center, Yorktown Heights, NY 10598 USA (e-mail: sanjeebd@us.ibm.com).

R. G. Baraniuk is with the Department of Electrical and Computer Engineering, Rice University, Houston, TX 77005-1892 USA (e-mail: richb@ rice.edu).

Digital Object Identifier 10.1109/TIP.2005.864171 compresses by quantizing the discrete cosine transform (DCT) coefficients of the image's three color planes; see Fig. 1 for an overview. However, the various settings employed during JPEG compression and decompression are not standardized [3]. The following JPEG settings can be chosen by the user or an imaging device:

1) the color space used to compress the image's three color planes independently;

2) the subsampling employed on each color plane during compression and the complementary interpolation employed during decompression;

3) the quantization table used to compress each color plane.

We refer to these settings as the image's JPEG compression history $(\mathrm{CH})$.

An image's $\mathrm{CH}$ is often not directly available from its current representation. For example, JPEG images are often imported into Microsoft Powerpoint or Word documents using graphics programs such as Microsoft Clip Gallery and then stored internally using a decompressed format. JPEG images are also routinely converted to lossless compression formats such as Windows bitmap (BMP) format (say, to create a background image for Windows or to feed a print driver) or Tagged Image File Format (TIFF). In such cases, the JPEG compression settings are discarded after decompression.

We aim to estimate the JPEG CH from a given JPEG-decompressed color image. We refer to this problem as JPEG Compression History Estimation (CHEst).

The $\mathrm{CH}$, if available, can be used for a variety of applications. The file-size of a JPEG image is typically significantly smaller than the file-size after the image's conversion to BMP or TIFF format. The JPEG CH enables us to effectively recompress such converted BMP and TIFF images; JPEG-compressing the image with previous JPEG settings yields significant file-size reduction without introducing additional distortion. The JPEG $\mathrm{CH}$ can also be used by "smart" print servers to reduce artifacts from received BMP images such as blocking and ringing due to previous JPEG compression. To alleviate such artifacts by adapting techniques described in [4] and [5], the print server would need the image's JPEG CH. An image's JPEG CH can also potentially be used as an authentication feature, for covert messaging, or to uncover the compression settings used inside digital cameras.

The CHEst problem is relatively unexplored. Fan and de Queiroz proposed a statistical framework to perform CHEst for gray-scale images [6]; for a gray-scale image, the $\mathrm{CH}$ comprises only the quantization table employed during previous JPEG 


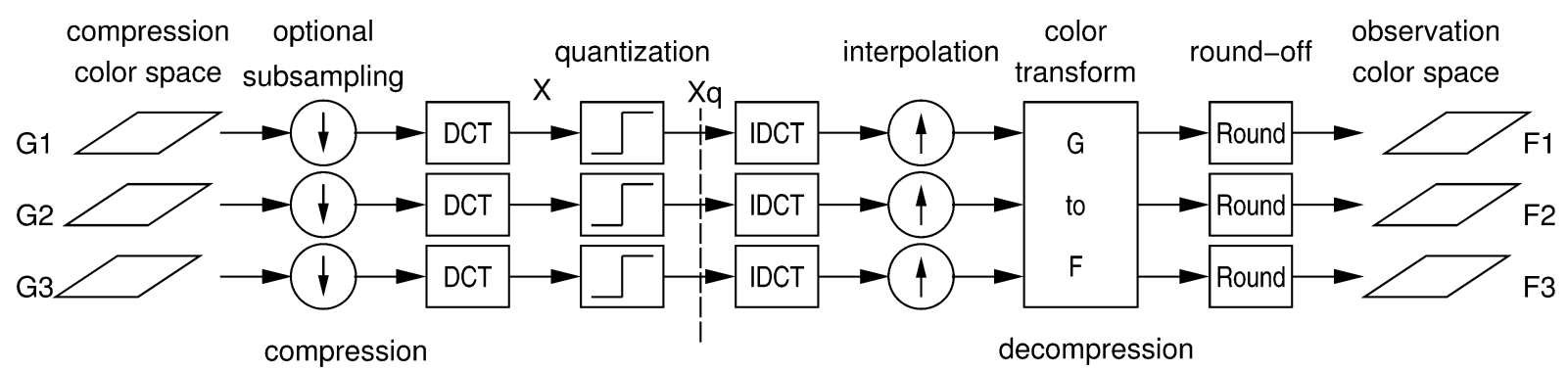

Fig. 1. Overview of JPEG compression and decompression.

operations. However, CHEst for color images remains unexplored. This paper proposes two new frameworks to perform CHEst for color images.

First, we derive a statistical framework for CHEst. We observe that JPEG leaves its signature by quantizing the image's DCT coefficients and forcing them to conform to near-periodic structures. We statistically characterize this near-periodicity for a single color plane. The resulting framework can be exploited to estimate a gray-scale image's $\mathrm{CH}$, namely, its quantization table. We extend the statistical framework to color images and design a dictionary-based CHEst approach. The dictionary consists of typical color transformations, subsampling factors, and interpolations. We adopt a maximum a posteriori (MAP) approach to estimate the color image's $\mathrm{CH}$ from the dictionary

$$
\{\widehat{G}, \widehat{S}, \widehat{Q}\}=\arg \max _{G, S, Q} P(\text { Image }, G, S, Q)
$$

with $P(\cdot)$ denoting the probability and $\widehat{G}, \widehat{S}, \widehat{Q}$ the estimated compression color space, subsampling and associated interpolation, and quantization tables, respectively.

Second, we consider the case when the transform from the color space used to perform quantization to the image's current representation color space is affine and when no subsampling is employed during JPEG compression. For such a case, we develop a novel, blind, lattice-based CHEst algorithm. Such a blind approach is required when an unknown proprietary color transform is employed by JPEG. We demonstrate that after JPEG decompression, such an image's DCT coefficients closely conform to a 3-D parallelepiped ${ }^{1}$ lattice structure determined by the affine color transform. Formally, a lattice is a set of integer linear combinations of a given set of vectors. The minimal set of vectors whose integer linear combinations span all lattice points is a lattice basis. We also demonstrate that the JPEG CH information is encoded in the nearly orthogonal bases that span the DCT lattices. Recently, [7] and [8] derived the geometric conditions for a lattice basis to contain the shortest nonzero lattice vector and the conditions to characterize such bases' uniqueness. Using these recent insights, and using novel applications of existing lattice algorithms, we estimate the color image's $\mathrm{CH}$, namely, the affine color transform and the quantization tables.

The proposed CHEst algorithms demonstrate excellent performance in practice. Further, we verify that CHEst allows us to recompress an image with minimal distortion [large signal-tonoise-ratio (SNR)] and simultaneously achieve a small file-size (see Figs. 7 and 8).

\footnotetext{
${ }^{1} \mathrm{~A}$ solid with six faces, each of which is a parallelogram.
}

The rest of this paper is organized as follows. We first provide a brief overview of color transforms and JPEG in Sections II and III. We derive the statistical CHEst framework for gray-scale images in Section IV and extend this framework to design dictionary-based CHEst for color images in Section V. In Section VI, we describe the 3-D lattice structure of a JPEG-decompressed image when JPEG uses an affine color transform and no subsampling. Section VII overviews the properties of nearly orthogonal lattice bases and some celebrated CHEst-relevant lattice problems. In Section VIII, we describe lattice-based CHEst and its experimental performance. We demonstrate CHEst's utility in JPEG recompression in Section IX and conclude in Section X.

\section{COLOR SPACES AND TRANSFORMS}

Color perception is a sensation produced when light excites the receptors in the human retina. Color can be described by specifying the light's spectral power distribution. Such a description is highly redundant because the human retina has only three types of receptors that influence color perception. ${ }^{2}$ Consequently, three numerical components are sufficient to describe a color; this is termed the trichromatic theory [2].

Based on the trichromatic theory, digital color imaging devices use three parameters to specify any color; the three parameters can be viewed as a 3-D vector. The color space is the reference coordinate system with respect to which the 3 -D vector describes color [1], [2]. There exist many different coordinate systems or color spaces according to which a color can be specified. For example, the Commission Internationale de L'Éclairage (CIE) defined the CIE $X Y Z$ color space to specify all visible colors using positive $X, Y$, and $Z$ values [1], [2]. Other examples include different varieties of $R G B$ (Red $R$, Green $G$, and Blue $B$ ) and $\mathrm{YCbCr}$ (luminance $Y$, and chrominances $\mathrm{Cb}$ and $\mathrm{Cr}$ ) color spaces. These color spaces are related to each other and to reference color spaces such as the $C I E X Y Z$ via linear or nonlinear color transformations. For example, the popular Independent JPEG Group (IJG) JPEG implementation [9] converts the digital color image's 0-255-valued $R, G, B$ components to 0-255-valued $\mathrm{Y}, \mathrm{Cb}, \mathrm{Cr}$ components using the following transformation:

$$
\left[\begin{array}{l}
Y \\
C b \\
C r
\end{array}\right]=\left[\begin{array}{ccc}
0.299 & 0.587 & 0.114 \\
-0.169 & -0.331 & 0.5 \\
0.5 & -0.419 & -0.081
\end{array}\right]\left[\begin{array}{l}
R \\
G \\
B
\end{array}\right]+\left[\begin{array}{c}
0 \\
128 \\
128
\end{array}\right]_{(2)}
$$

\footnotetext{
${ }^{2} \mathrm{~A}$ fourth type of receptor is also present in the retina, but it does not affect color perception because it is effective only at extremely low light levels [2].
} 


$\begin{array}{cccccccc}10 & 7 & 6 & 10 & 14 & 24 & 31 & 37 \\ 7 & 7 & 8 & 11 & 16 & 35 & 36 & 33 \\ 8 & 8 & 10 & 14 & 24 & 34 & 41 & 34 \\ 8 & 10 & 13 & 17 & 31 & 52 & 48 & 37 \\ 11 & 13 & 22 & 34 & 41 & 65 & 62 & 46 \\ 14 & 21 & 33 & 38 & 49 & 62 & 68 & 55 \\ 29 & 38 & 47 & 52 & 62 & 73 & 72 & 61 \\ 43 & 55 & 57 & 59 & 67 & 60 & 62 & 59\end{array}$

Quantization table 1

$\begin{array}{llllllll}10 & 11 & 14 & 28 & 59 & 59 & 59 & 59 \\ 11 & 13 & 16 & 40 & 59 & 59 & 59 & 59 \\ 14 & 16 & 34 & 59 & 59 & 59 & 59 & 59 \\ 28 & 40 & 59 & 59 & 59 & 59 & 59 & 59 \\ 59 & 59 & 59 & 59 & 59 & 59 & 59 & 59 \\ 59 & 59 & 59 & 59 & 59 & 59 & 59 & 59 \\ 59 & 59 & 59 & 59 & 59 & 59 & 59 & 59 \\ 59 & 59 & 59 & 59 & 59 & 59 & 59 & 59\end{array}$

Quantization table 2

Fig. 2. Examples of JPEG quantization tables for $8 \times 8$ DCT blocks.

The resulting $\mathrm{YCbCr}$ space is also referred to as the ITU.BT-601 $\mathrm{YCbCr}$ space [1]. The inverse color transformation from the ITU.BT-601 $\mathrm{YCbCr}$ space to the $R G B$ space is given by

$$
\left[\begin{array}{l}
R \\
G \\
B
\end{array}\right]=\left[\begin{array}{ccc}
1.0 & 0.0 & 1.402 \\
1.0 & -0.344 & -0.714 \\
1.0 & 1.772 & 0.0
\end{array}\right]\left(\left[\begin{array}{c}
Y \\
C b \\
C r
\end{array}\right]-\left[\begin{array}{c}
0 \\
128 \\
128
\end{array}\right]\right) .
$$

The transforms in both (2) and (3) are affine. Henceforth, we refer to the $3 \times 3$ matrix as the affine transform's linear component and the $3 \times 1$ shift as the affine transform's additive component.

Later in this paper, we will invoke a variety of color spaces that are inter-related by affine or nonlinear transforms. We refer the reader to [1] and [2] for additional information on color, different color spaces, and transforms.

\section{EFFECTS OF JPEG COMPRESSION AND DECOMPRESSION}

In this section, we review the CHEst-relevant JPEG compression and decompression steps. We do not describe all JPEG operations, but present a model that fully accounts for the effects of JPEG compression and decompression on an image. The model folds quantization and dequantization into one step, and ignores all entropy coding steps because these do not affect the final image. For further JPEG details, we refer the reader to [3].

Consider an observed color image represented in the hypothetical $F$ color space (see Fig. 1); $F 1, F 2$, and $F 3$ denote the three color planes. We refer to the $F$ space as the observation color space. Assume that the image was previously JPEG-compressed in the $G$ color space-termed the compression color space.

JPEG compression essentially performs the following operations independently on each color plane $G 1, G 2$, and $G 3$ in the $G$ space.

1) Optionally downsample each color plane (for example, retain alternate pixels to downsample by a factor of two); this process is termed subsampling.

2) Split each color plane into nonoverlapping $8 \times 8$ blocks. Take the two-dimensional (2-D) DCT of each block.

3) Quantize the coefficients at each DCT frequency to the closest integer multiple of the quantization step-size corresponding to that frequency. For example, if $X$ denotes an arbitrary DCT coefficient and $q$ the quantization stepsize for the corresponding DCT frequency, then the quantized DCT coefficient $\bar{X}_{q}$ is obtained by

$$
\bar{X}_{q}:=\operatorname{round}\left(\frac{X}{q}\right) q \text {. }
$$

See Fig. 2 for examples of quantization tables; each entry in the $8 \times 8$ quantization table is the quantization step-size for an $8 \times 8$ image block's corresponding DCT coefficient.

JPEG decompression performs the following operations.

1) Compute the inverse DCTs of the $8 \times 8$ blocks of quantized coefficients.

2) Interpolate the downsampled color planes by repetition followed by optional spatial smoothing with a low-pass filter. The popular IJG JPEG implementation [9] uses a $1 / 4 \times\left[\begin{array}{lll}1 & 2 & 1\end{array}\right]$ impulse response filter to smooth in the horizontal and vertical directions.

3) Transform the decompressed image to the desired color space $F$ using the appropriate $G$ to $F$ transformation.

4) Round-off resulting pixel values to the nearest integer so that they lie in the $0-255$ range. ${ }^{3}$

Henceforth, we will refer to the zero frequency DCT coefficient as the $d c$ coefficient and the remaining 63 DCT coefficients as the $a c$ coefficients.

\section{CHEST FOR GRAY-SCALE IMAGES}

For gray-scale images, JPEG compression and decompression replicates the steps outlined in Section III for a single color plane but without subsampling and interpolation. Hence, the CHEst problem simplifies to estimating the quantization tables employed during the previous JPEG compression. Due to JPEG's quantization operations, the JPEG-decompressed gray-scale images' DCT coefficient histograms exhibit a near-periodic structure with the period determined by the quantization step-size. In this section, to estimate the quantization table, we derive a statistical framework that characterizes the near-periodic structure.

\section{A. Statistical Framework}

An arbitrary DCT coefficient $\widetilde{X}$ of a JPEG-decompressed gray-scale image can be obtained by adding to the corresponding quantized coefficient $\bar{X}_{q}$ [see (4)] a round-off error term $\Gamma$

$$
\tilde{X}=\bar{X}_{q}+\Gamma
$$

${ }^{3}$ In reality, round-offs occur also after the inverse DCT step. The model consolidates all the round-off operations into one step for the sake of simplicity. 
As described in [6], we can model $\Gamma$ using a truncated Gaussian distribution

$$
P(\Gamma=t)=\Upsilon \exp \left(-\frac{t^{2}}{2 \sigma^{2}}\right), \quad \text { for } t \in[-\zeta, \zeta]
$$

with $\sigma^{2}$ the Gaussian's variance, $[-\zeta, \zeta]$ the truncated Gaussian's support, and $\Upsilon$ the normalizing constant. For example, $\sigma^{2}=0.8$ and $\zeta=6$. Further, based on studies in [3], [10], we can model the DCT coefficients using a zero-mean Laplacian distribution

$$
P(X=t)=\frac{\lambda}{2} \exp (-\lambda|t|) .
$$

We have assumed that the parameter $\lambda$ is known; in practice, we estimate $\lambda$ from the observed decompressed image for each DCT frequency as described later in this section. From (7), we have

$$
L_{\lambda}(k q):=P\left(\bar{X}_{q}=k q \mid q, k \in \mathbb{Z}\right)=\int_{(k-0.5) q}^{(k+0.5) q} \frac{\lambda}{2} \exp (-\lambda|\tau|) d \tau
$$

and hence

$$
P\left(\bar{X}_{q}=t \mid q\right)=\sum_{k \in \mathbb{Z}} \delta(t-k q) L_{\lambda}(k q) .
$$

Now, assuming that the round-off error $\Gamma$ is independent of $X$ and $q, \widetilde{X}$ 's distribution is obtained by convolving the distributions for $\bar{X}$ and $\Gamma$ (see Fig. 3); that is

$$
\begin{aligned}
& P(\tilde{X}=t \mid q) \\
& \quad=\int P\left(\bar{X}_{q}=\tau \mid q\right) P(\Gamma=t-\tau) d \tau \\
& \quad= \begin{cases}\sum_{k \in \mathbb{Z}} \Upsilon \exp \left(-\frac{|t-k q|^{2}}{2 \sigma^{2}}\right) L_{\lambda}(k q), & \text { for }|t-k q| \in[-\zeta, \zeta] \\
0, & \text { otherwise. }\end{cases}
\end{aligned}
$$

Let $\mathcal{D}_{i}$ denote the set of the JPEG-decompressed image's $i$ th-frequency DCT coefficients; that is, $\mathcal{D}_{i}$ comprises one coefficient from each $8 \times 8$ block. Given $\mathcal{D}_{i}$, we can obtain the MAP estimate $\widehat{q}_{i}$ of the quantization step used on the $i$ th-frequency coefficients during previous compression as

$$
\begin{aligned}
\widehat{q}_{i} & =\arg \max _{q \in \mathbb{Z}^{+}} P\left(\mathcal{D}_{i}, q\right) \\
& =\arg \max _{q \in \mathbb{Z}^{+}}\left(\prod_{\widetilde{X} \in \mathcal{D}_{i}} P(\widetilde{X} \mid q) P(q)\right)
\end{aligned}
$$

where the DCT coefficients are assumed to be independent, and $P(q)$ denotes the prior on $q$.

\section{B. Algorithm Steps}

Using the statistical framework derived in the previous section, we can estimate the $8 \times 8$ quantization table $Q:=\left\{q_{i}\right\}$, with $i=1, \ldots, 64$, enumerating the $64 \mathrm{DCT}$ frequencies, using the following steps.

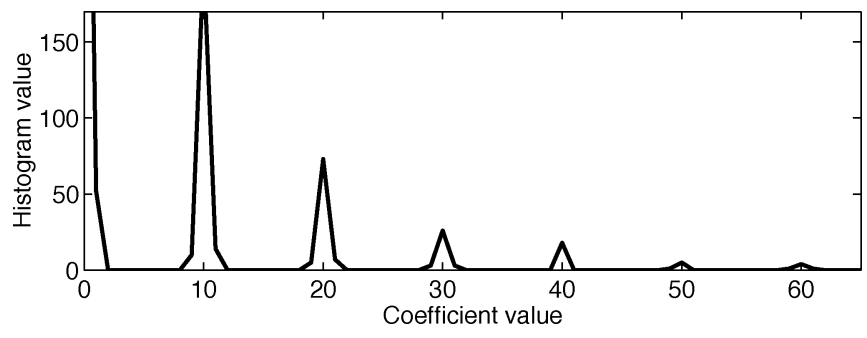

Fig. 3. Histogram of quantized DCT coefficients. The DCT coefficients from DCT frequency $(4,4)$ of the gray-scale Lena image were subjected to quantization with step-size $q=10$ during JPEG compression and then decompressed. Due to roundoff errors, the DCT coefficients are perturbed from integer multiples of 10 .

1) For each frequency $i$, compute the set $\mathcal{D}_{i}$ of the observed decompressed image's DCT coefficients.

2) Estimate the parameter $\lambda$ from the observations as

$$
\lambda=\frac{N}{\sum_{\widetilde{X} \in \mathcal{D}_{i}}|\widetilde{X}|}
$$

with $N$ the number of coefficients in the set $\mathcal{D}_{i}$.

3) Assuming a uniform prior on $q$, use (11) with suitable parameters $\sigma^{2}$ and $\zeta$ to estimate

$$
\widehat{q}_{i}=\arg \max _{q \in \mathbb{Z}^{+}}\left(\prod_{\widetilde{X} \in \mathcal{D}_{i}} P(\widetilde{X} \mid q)\right) .
$$

This algorithm is not entirely new; it is a refinement of the technique proposed by Fan and de Queiroz in [6]. While the core ideas remain the same, the final derived (11) differs because of significant variations in the starting points for the derivation and in the intermediate assumptions. Further, our derivation explicitly accounts for all normalization constants, thereby allowing us to extend the above approach to estimate the $\mathrm{CH}$ of color images.

\section{Dictionary-BASED CHEST FOR COLOR IMAGES}

In this section, we build on the quantization step-size estimation algorithm for gray-scale images from Section IV to perform CHEst for color images.

\section{A. Statistical Framework}

For color images, in addition to quantization, JPEG performs color transformation and subsampling along with the complementary interpolation. We observe that the DCT coefficient histogram of each color plane exhibits the near-periodic structure of Fig. 3 introduced by quantization only when the image is transformed to the original compression color space and all interpolation artifacts are removed. Hence, we can obtain the MAP estimate of a color image's $\mathrm{CH}$ as in (1) using a simple extension of the statistical framework for gray-scale images.

Let $\widetilde{X}_{G j, S, i}$ denote the set of $i$ th-frequency DCT coefficients from the $j$ th-color plane, $j=1,2,3$. Assume that $\widetilde{X}_{G j, S, i}$ is obtained by first transforming the image from the $F$ to the $G$ color 
space representation, then undoing the interpolation $S$, and finally taking the DCT of the color planes. Let $\mathcal{D}_{G j, S, i}$ denote the set of all $\widetilde{X}_{G j, S, i}$. Then

$$
\begin{aligned}
& \{\widehat{G}, \widehat{S}, \widehat{Q}\} \\
& =\arg \max _{G, S, Q} P(\text { Image } \mid G, S, Q) P(G, S, Q) \\
& =\arg \max _{G, S, Q} \prod_{i=1}^{64} \prod_{j=1}^{3} P\left(\mathcal{D}_{G j, S, i} \mid G, S, Q\right) P(G) P(S) P(Q) \\
& =\arg \max _{G, S, Q} \prod_{i=1}^{64} \prod_{j=1}^{3} \prod_{\widetilde{X}_{G, S} \in \mathcal{D}_{G, S}} P\left(\widetilde{X}_{G j, S, i} \mid G, S, Q\right) \\
& \quad \times P(G) P(S) P(Q)
\end{aligned}
$$

assuming that all the $\widetilde{X}_{G j, S, i}$ coefficients and the choices of $G, S$, and $Q$ are independent. In (15), the DCT coefficients' conditional probability $P\left(\widetilde{X}_{G j, S, i} \mid G, S, Q\right)$ is computed using (11), which is a metric for how well the image DCT coefficients conform to a near-periodic structure. Hence, if $G, S$, and $Q$ were actually employed during the previous JPEG compression, then the histogram of the $i$ th-frequency DCT coefficients would be nearly periodic, and the associated $P\left(\widetilde{X}_{G j, S, i} \mid G, S, Q\right)$ would be large. Consequently, the MAP estimate would be accurate.

\section{B. Algorithm Steps}

In general, the MAP estimation in (15) would require a search over all $G$ and $S$. For practical considerations, we constrain the search to a dictionary comprising commonly employed compression color spaces and interpolations. Dictionary-based CHEst steps are as follows.

1) Choose a test color space $G$ and interpolation method $S$ from the dictionary.

2) Transform the observed color image to the color space $G$.

3) Undo the effects of the test interpolation $S$. To undo interpolation by simple repetition, simply downsample the color plane. To undo interpolation by repetition and smoothing, first deconvolve the smoothing using a simple Tikhonov-regularized deconvolution filter [11] and then downsample the color plane.

4) Employ the quantization table estimation step from Section IV on each color plane.

5) Try all the $G$ and $S$ in the dictionary, and output the $G$ and $S$ yielding the maximum conditional probability (15) along with the associated quantization tables from Step 4.

Dictionary-based CHEst's computational complexity is determined by the image size, the number of the test color spaces, and the number of test subsamplings and interpolations in the dictionary. In practice, a majority of the dictionary elements could be easily and reliably eliminated using just a small part of the image. The $\mathrm{CH}$ can then be estimated quickly by applying the dictionary-based CHEst with the pruned dictionary on the entire image.

\section{Dictionary-Based CHEst Results}

Dictionary-based CHEst precisely estimates a JPEG-decompressed color image's $\mathrm{CH}$ when the dictionary contains the actual color transform and interpolation. We demonstrate dictionary-based CHEst's performance using the $512 \times 512$ Lena color image [12] and a specific JPEG CH choice. The algorithm performed equally well on a wide variety of experiments comprising different images and compression color spaces. The Matlab scripts to recreate these results can be downloaded from www.dsp.rice.edu/software.

We JPEG-compressed Lena in the 8-bit CIELab color space using the $s R G B$ to 8-bit CIELab color transformation [1] and employed $2 \times 2,1 \times 1,1 \times 1$ subsampling; that is, the luminance $L$ color plane was not downsampled, while the chrominance planes $a$ and $b$ were downsampled by a factor of 2 in the horizontal and vertical directions. We employed quantization tables 1 from Fig. 2 for the $L$ plane and quantization table 2 from Fig. 2 for the both the $a$ and $b$ planes. During decompression, the $a$ and $b$ planes were interpolated by first upsampling using repetition and then smoothing in the horizontal and vertical di-

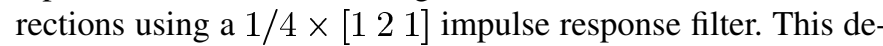
compressed image is the input to dictionary-based CHEst.

To perform CHEst, we tested all color transforms from a dictionary consisting of $R G B$ to ITU.BT-601 YCbCr, Computer RGB to ITU.BT-601 YCbCr, Studio RGB to ITU.BT-601 YCbCr, RGB to Kodak PhotoYCC, sRGB to Linear RGB, sRGB to 8-bit CIELab, and $s R G B$ to $C M Y$ transforms [1]. For each transform, we considered subsampling factors $2 \times 2,1 \times 1,1 \times 1$ (with and without smoothing during interpolation) and $1 \times 1,1 \times 1,1 \times 1$.

During the conditional probability computations (15), we assumed that all color transforms and quantization step-sizes are equally likely; that is, we set $P(G)=P(Q)=1$. When larger subsampling factors and smoothing are employed, the DCT coefficients deviate further from their quantized values, resulting in relatively lower conditional probabilities. To level this effect, we need to adapt $\sigma^{2}$ and the priors $P(S)$. To test if a color plane was subsampled by a factor of 2 and then smoothed during interpolation, we set the $\sigma^{2}=0.8$ [see (11)] during the quantization table estimation step. To test if no smoothing was employed during interpolation, we set $\sigma^{2}=0.75$, and to test if no subsampling was employed, we reduced the $\sigma^{2}$ to 0.5 . Further, we set the prior $P(S)=0.55$ for the $2 \times 2,1 \times 1,1 \times 1$ with smoothing, $P(S)=0.35$ for the $2 \times 2,1 \times 1,1 \times 1$ without smoothing, and $P(S)=0.1$ for the $1 \times 1,1 \times 1,1 \times 1$ subsampling. We set $\zeta=6$ in (11) during our experiments. These settings worked well on all our experimental tests.

By comparing the conditional probabilities' natural logarithms (listed in Table I), we precisely identified that the $s R G B$ to 8-bit CIELab color transformation was employed with $2 \times$ $2,1 \times 1,1 \times 1$ subsampling during the previous compression, and that smoothing was employed during the decompression; the corresponding conditional probability value (enclosed by a $\square$ in Table I) is the largest.

Fig. 4 illustrates the algorithm's quantization table estimates (see Fig. 2 for the actual tables). Our quantization step-size estimates were quite accurate, especially at the more important low frequencies. Note that to estimate the quantization step-size, at least one coefficient should be quantized to a nonzero value. For many of the high frequencies, all coefficients were quantized to zero because the actual quantization step-sizes (see Fig. 2) were large. For such quantized-to-zero frequencies, our algorithm typically returned the maximum quantization step size included in our search range (100 in our case); they are marked by 
TABLE I

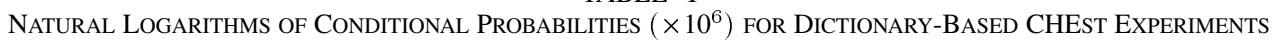

\begin{tabular}{|c|c|c|c|}
\hline Color transform & $1 \times 1,1 \times 1,1 \times 1$ & $\begin{array}{c}2 \times 2,1 \times 1,1 \times 1 \\
\text { (without smoothing) }\end{array}$ & $\begin{array}{c}2 \times 2,1 \times 1,1 \times 1 \\
\text { (with smoothing ) }\end{array}$ \\
\hline RGB to ITU.BT-601 YCbCr & -0.88 & -0.88 & -0.8 \\
Computer RGB to ITU.BT-601 YCbCr & -0.83 & -0.83 & -0.75 \\
Studio RGB to ITU.BT-601 YCbCr & -0.88 & -0.89 & -0.81 \\
$R G B$ to Kodak PhotoYCC & -0.79 & -0.78 & -0.69 \\
sRGB to Linear RGB & -1.5 & -1.9 & -1.8 \\
sRGB to 8-bit CIELab & -0.73 & -0.71 & $-\mathbf{0 . 5 3}$ \\
sRGB to CMY & -1.5 & -1.9 & -1.8 \\
\hline
\end{tabular}

$\begin{array}{cccccccc}10 & 7 & 6 & 10 & 14 & 24 & 31 & 37 \\ 7 & 7 & 8 & 11 & 16 & 35 & 36 & 32 \\ 8 & 8 & 10 & 14 & 24 & 34 & 40 & 33 \\ 8 & 10 & 13 & 17 & 31 & 51 & 47 & 35 \\ 11 & 13 & 22 & 34 & 40 & 63 & \times & 44 \\ 14 & 21 & 32 & 37 & 47 & \times & \times & 51 \\ 28 & 35 & 44 & 49 & \times & \times & \times & \times \\ \times & \times & \times & \times & \times & \times & \times & \times\end{array}$

$L$ 's table
Fig. 4. Dictionary-based CHEst algorithm's quantization tables estimates for the 8-bit CIELab's $L, a$, and $b$ color planes.

$\times$ 's in Fig. 4. For some quantized-to-zero frequencies in the $a$ and $b$ planes, our algorithm did not return the maximum quantization step-size because the $a$ and $b$ plane coefficients are more noisy (compared to the $L$ plane coefficients) due to the additional deconvolution step (Step 3 in Section V-B). For example, our algorithm estimated one of the $a$ plane's quantization step-sizes to be 7 , whereas the actual quantization step-size was 59 . Note that such errors, though seemingly large, have negligible impact on applications such as recompression; any quantization step-size estimate greater than 1 would reset most of the DCT coefficients to zero (as desired) during recompression.

\section{BLIND CHEST AND LATTICES}

The dictionary-based CHEst approach described in Section V would fail if an unknown proprietary color space was used to perform the JPEG compression. This motivates us to develop a blind approach that does not rely on a fixed dictionary of known color spaces. Blind lattice-based CHEst can handle cases where the transform from the compression color space to the current color space is affine and no subsampling is employed during the previous JPEG compression. For such cases, Blind CHEst aims to estimate the affine transform and quantization tables employed during the previous JPEG compression from the JPEG-decompressed image. The lattice geometry of a JPEG decompressed image's DCT coefficients holds the key to blind JPEG CHEst.

\section{A. Lattice Fundamentals}

Lattices are central to a number of fields including coding theory, number theory, and cryptography [13]-[16]. A lattice is the set of all linear integer combinations of a finite set of vectors. In $\mathbb{R}^{n}$, a lattice $\mathcal{L}$ is defined as the set $\left\{\mathcal{B} u: u \in \mathbb{Z}^{m}\right\}$, with $\mathcal{B}$ a real $n \times m$ matrix. Figs. 5(a) and (b) are both illustrations of 3 -D lattices. The columns of $\mathcal{B}$ are said to span the lattice $\mathcal{L}$. If $\mathcal{B}$ contains the minimal set of vectors spanning $\mathcal{L}$, then it is termed a basis for $\mathcal{L}$. A lattice can have more than one basis. Any two bases $\mathcal{B}_{1}$ and $\mathcal{B}_{2}$ for $\mathcal{L}$ have the same number of vectors and are related by $\mathcal{B}_{1}=\mathcal{B}_{2} \mathcal{U}$, with $\mathcal{U}$ an unimodular matrix - an integer matrix with determinant equal to \pm 1 .

\section{B. Ideal Lattice Structure of DCT Coefficients}

In the absence of round-off noise, due to JPEG's quantization step, a JPEG-decompressed color image's 3-D DCT vectors conform to a regular parallelepiped lattice structure.

Consider an arbitrary $8 \times 8$ uncompressed color image block in the $G$ color space that the DCT acts on during JPEG compression. Let $X_{G 1, i}, X_{G 2, i}$, and $X_{G 3, i}$ denote the respective $i$ th-frequency DCT coefficients of the $G 1, G 2$, and $G 3$ planes in the chosen $8 \times$ 8 color image block. As described in Section III, JPEG quantizes each plane's DCT coefficients independently to

$$
\begin{aligned}
\bar{X}_{G, i} & :=\left[\begin{array}{l}
\bar{X}_{G 1, q_{i, 1}} \\
\bar{X}_{G 2, q_{i, 2}} \\
\bar{X}_{G 3, q_{i, 3}}
\end{array}\right] \\
& :=\left[\begin{array}{ccc}
q_{i, 1} & 0 & 0 \\
0 & q_{i, 2} & 0 \\
0 & 0 & q_{i, 3}
\end{array}\right]\left[\begin{array}{l}
\operatorname{round}\left(\frac{X_{G 1, i}}{q_{i, 1}}\right) \\
\operatorname{round}\left(\frac{X_{G 2, i}}{q_{i, 2}}\right) \\
\operatorname{round}\left(\frac{X_{G 3, i}}{q_{i, 3}}\right)
\end{array}\right] \\
:= & {\left[\begin{array}{c}
\operatorname{round}\left(\frac{X_{G 1, i}}{q_{i, 1}}\right) \\
\operatorname{round}\left(\frac{X_{G 2, i}}{q_{i, 2}}\right) \\
\operatorname{round}\left(\frac{X_{G 3, i}}{q_{i, 3}}\right)
\end{array}\right] }
\end{aligned}
$$




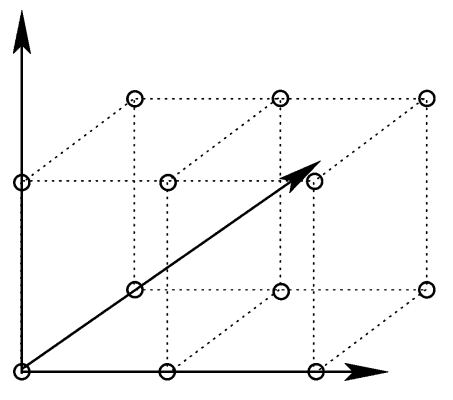

(a) G space

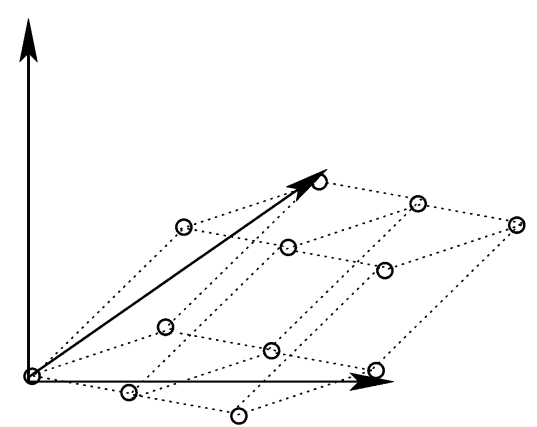

(b) F space (no round-off)

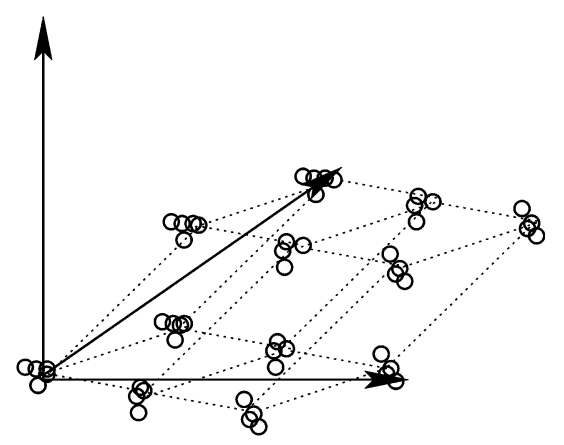

(c) F space (with round-off)

Fig. 5. Lattice structures in a JPEG-decompressed color image. (a) In the $G$ space, all 3-D DCT vectors from the same DCT frequency lie on a rectangular lattice's vertices. The 3-D vectors are denoted by small circles. (b) Assuming round-off errors are absent, in the $F$ space, the 3-D DCT vectors lie on a parallelepiped lattice's vertices. (c) Round-off errors slightly perturb the $F$ space 3-D DCT vectors from the parallelepiped lattice locations.

with $q_{i, 1}, q_{i, 2}$, and $q_{i, 3}$ the respective quantization step-sizes for the three color planes. Clearly, since $\left[\operatorname{round}\left(X_{G 1, i} / q_{i, 1}\right), \operatorname{round}\left(X_{G 2, i} / q_{i, 2}\right), \operatorname{round}\left(X_{G 3, i} / q_{i, 3}\right)\right]^{T}$ belongs to $\mathbb{Z}^{3}$ (the superscript $T$ denotes matrix transpose), the vector $\left[\bar{X}_{q_{i, 1}}, \bar{X}_{q_{i, 2}}, \bar{X}_{q_{i, 3}}\right]^{T}$ lies on a 3-D lattice with basis $\mathcal{Q}_{i}$. Fig. 5(a) illustrates that the quantized 3-D DCT vector lies on a 3-D lattice; it is a rectangular box lattice because $\mathcal{Q}_{i}$ 's columns are orthogonal to each other.

After quantization, assume that the image is subjected to an affine color transform from the $G$ to the $F$ color space. Let $\mathcal{T}$ denote the affine transform's linear component. Further assume that no round-off is performed. Then, the transformed image's $i$ th-frequency $(i \neq \mathrm{dc}) 3-\mathrm{D}$ DCT vectors can be expressed as

$$
\begin{aligned}
X_{F, i} & :=\left[\begin{array}{l}
X_{F 1, i} \\
X_{F 2, i} \\
X_{F 3, i}
\end{array}\right] \\
& :=\mathcal{T}\left[\begin{array}{l}
\bar{X}_{G 1, q_{i, 1}} \\
\bar{X}_{G 2, q_{i, 2}} \\
\bar{X}_{G 3, q_{i, 3}}
\end{array}\right]=\mathcal{T} \mathcal{Q}_{i}\left[\begin{array}{l}
\operatorname{round}\left(\frac{X_{G 1, i}}{q_{i, 1}}\right) \\
\operatorname{round}\left(\frac{X_{G 2, i}}{q_{i, 2}}\right) \\
\operatorname{round}\left(\frac{X_{G 3, i}}{q_{i, 3}}\right)
\end{array}\right] .
\end{aligned}
$$

Thus, in the $F$ space representation, the $i$ th-frequency 3-D DCT vectors lie on a lattice $\mathcal{L}_{i}$ with basis $\mathcal{T} \mathcal{Q}_{i}$. (The affine transform's additive component affects only the dc coefficients; it shifts the dc coefficient lattice away from the origin.) Fig. 5(b) illustrates the lattice geometry of the 3-D DCT vectors in the $F$ space; the vectors lie on a parallelepiped lattice because $\mathcal{T} \mathcal{Q}_{i}$ 's columns are not orthogonal.

\section{Round-Offs Perturb Ideal Lattice Geometry}

In reality, a JPEG-decompressed image is always subjected to round-off during the decompression (see Fig. 1 and Section III). Hence, any 3-D DCT vector of the given JPEG-decompressed image can be expressed as

$$
\widetilde{X}_{F, i}=X_{F, i}+N_{i}
$$

with $N_{i}$ denoting the 3-D round-off noise vector. Based on (6), we can statistically model $N_{i}$ as

$$
P\left(\left\|N_{i}\right\|_{2}=t\right)=\Upsilon \exp \left(-\frac{t^{2}}{2 \sigma^{2}}\right), \quad \text { for } t \in[-\zeta, \zeta] .
$$

Thus, from (18), the 3-D DCT vectors in the $F$ color space lie approximately on a parallelepiped lattice $\mathcal{L}_{i}$ [see Fig. 5(c)]. (The dc coefficients lie approximately on a parallelepiped structure that is shifted from the origin.)

\section{Blind CHEst and Nearly Orthogonal Bases}

A JPEG-decompressed image's $i$ th-frequency $(i \neq \mathrm{dc}) 3-\mathrm{D}$ DCT vectors lie approximately on a lattice $\mathcal{L}_{i}$ with basis $\mathcal{T} \mathcal{Q}_{i}$. A key step in blind CHEst is to estimate $\mathcal{T} \mathcal{Q}_{i}$. However, since a lattice can have multiple bases, we must exploit some additional information about practical affine color transforms to resolve the basis ambiguity.

Practical affine color transforms simply try to find a shifted and approximately rotated reference coordinate system to describe color. Consequently, the linear component $\mathcal{T}$ (and thereby, $\mathcal{T} \mathcal{Q}_{i}$ ) of all practical affine color transforms will be "nearly orthogonal". (To be precise, we will assume that $\mathcal{T}$ is weakly $(\pi / 3)+\epsilon$-orthogonal; see Section VII-C for the definition.) Therefore, in addition to lattice algorithms, we will also need to understand the properties of nearly orthogonal lattice bases.

\section{LATTICES ALGORITHMS AND PROPERTIES OF NEARLY ORTHOGONAL BASES}

We briefly review some celebrated CHEst-relevant lattice problems and some recent results on nearly orthogonal bases.

\section{A. Lattice Reduction and the Lenstra-Lenstra-Lovasz (LLL) Algorithm}

A celebrated problem of interest to us is the lattice reduction problem, which can be stated as follows. Given a set of vectors $b_{i}$ 's that span a lattice $\mathcal{L}$, find an ordered set of basis vectors for $\mathcal{L}$ such that the basis vectors are nearly orthogonal, and the shorter basis vectors appear first in the ordering [17]. Lattice reduction is clearly relevant because we also seek nearly orthogonal lattice bases in CHEst.

A major breakthrough in lattice theory was the discovery of a polynomial time lattice reduction algorithm by Lenstra, Lenstra, and Lovasz [13]; this algorithm is commonly referred to as the LLL algorithm. The LLL algorithm can be intuitively understood as an adaptation of Gram-Schmidt orthogonalization [18] 
that sequentially processes the vectors $b_{i}$ and maintains a basis spanning the processed vectors. We will invoke LLL in Section VIII to estimate a nearly orthogonal basis that spans the DCT coefficient lattice.

\section{B. Closest Vector Problem (CVP) and Shortest Vector Problem (SVP)}

The CVP and the SVP are two other famous NP-hard CHEstrelevant lattice problems [14], [15], [19]. They are both closely related to the lattice reduction problem. The CVP aims to find the closest (in the Euclidean sense) lattice point to a given point. For a comprehensive semitutorial paper on the CVP and algorithms to solve it, we refer the reader to [14]. The SVP aims to find the shortest nonzero lattice point. The 3-D DCT vectors lie only approximately on a lattice (see Section VI-C). To estimate a basis that approximately span these perturbed DCT vectors, we will invoke CVP solutions in Section VIII-B1.

\section{Properties of Nearly Orthogonal Lattice Basis Vectors}

Recently, [7] and [8] quantified the "orthogonality" of a basis in terms of the angle between its constituent vectors. An ordered set of vectors $\left\{b_{1}, b_{2}, \ldots, b_{m}\right\}$ is weakly $\theta$-orthogonal if for any $i=2, \ldots, m$, the angle between $b_{i}$ and the subspace spanned by $\left\{b_{1}, \ldots, b_{i-1}\right\}$ lies in the range $[\theta, \pi / 2]$; that is

$$
\begin{aligned}
\cos ^{-1}\left(\frac{\left|\left\langle b_{i}, \sum_{j=1}^{i-1} \alpha_{i} b_{i}\right\rangle\right|}{\left\|b_{i}\right\|_{2}\left\|\sum_{j=1}^{i-1} \alpha_{i} b_{i}\right\|_{2}}\right) \geq \theta \\
\text { for all } \alpha_{j} \in \mathbb{R} \text { with } \sum_{j}\left|\alpha_{j}\right|>0 .
\end{aligned}
$$

For example, the ITU.BT-601 YCbCr to RGB transform's [see (3)] linear component is weakly $\theta$-orthogonal with $\theta=0.349 \pi$ radians.

Theorem 1: Any weakly $(\pi / 3)+\epsilon$-orthogonal, $0<\epsilon \leq \pi / 6$ basis contains every shortest nonzero lattice vector.

Theorem 1's proof, which is provided in [7] and [8], follows by induction.

The next theorem addresses the uniqueness of weakly $(\pi / 3)+\epsilon$-orthogonal lattice basis in $\mathbb{R}^{3}$.

Theorem 2: Let $\mathcal{B}_{1}=\left\{b_{1}, b_{2}, b_{3}\right\}$ and $\mathcal{B}_{2}=\left\{\widetilde{b}_{1}, \widetilde{b}_{2}, \widetilde{b}_{3}\right\}$ be two weakly $(\pi / 3)+\epsilon$-orthogonal bases for a lattice $\mathcal{L}$ in $\mathbb{R}^{3}$. Let $\mathcal{U}$ be a unimodular matrix such that $\mathcal{B}_{1}=\mathcal{B}_{2} \mathcal{U}$. Then, the $\mathcal{U}$ 's elements' absolute values are upper-bounded by

$$
\kappa\left(\mathcal{B}_{1}\right):=\frac{4}{3} \frac{\max _{j \in\{1,2,3\}}\left\|b_{j}\right\|_{2}}{\min _{j \in\{1,2,3\}}\left\|b_{j}\right\|_{2}} .
$$

See [8] for the proof and further details. Theorem 2 guarantees that in $\mathbb{R}^{3}$, a weakly orthogonal basis with nearly equal length vectors is related to every weakly orthogonal basis by a unimodular matrix with small elements. For example, if $\mathcal{B}_{1}$ is weakly $(\pi / 3)+\epsilon$-orthogonal and its column lengths are within a factor of 1.5 of each other, then the unimodular matrix elements relating $\mathcal{B}_{1}$ to another weakly $(\pi / 3)+\epsilon$-orthogonal basis $\mathcal{B}_{2}$ are either 0 or \pm 1 .

\section{BLIND LATTICE-BASED CHEST FOR COLOR IMAGES}

In Section VIII-A, we use ideas from Section VII to develop a blind CHEst approach. Section VIII-B specifies the modifications required to robustify blind CHEst to round-off noise.

\section{A. Lattice-Based CHEst in the Absence of Round-Off Noise}

Lattice-based CHEst employs the following steps to solve the blind CHEst problem. Each step's detail is described in the subsections that follow immediately.

1) Estimate weakly $\pi / 3$-orthogonal bases $\mathcal{B}_{i}$ 's for ac DCT coefficient lattices $\mathcal{L}_{i}$ 's using LLL.

2) Estimate the color transform's scaled linear component $\mathcal{T} \mathcal{Q}_{i}$ from the estimated $\mathcal{B}_{i}$ 's.

3) Separate the $\mathcal{T} \mathcal{Q}_{i}$ 's into $\mathcal{T}$ and $\mathcal{Q}_{i}$ 's.

4) Estimate the $\mathcal{Q}_{i}$ for the dc frequency and color transform's additive component.

We will assume that $\mathcal{T}$ is weakly $(\pi / 3)+\epsilon$-orthogonal, $0 \leq \epsilon \leq$ $\pi / 6$; see Section VI-D for this assumption's motivation. ${ }^{4}$ We have verified that all the affine color transforms in the literature [1] satisfy this assumption. ${ }^{5}$

1) Estimating $\pi / 3$-Orthogonal Bases $\mathcal{B}_{i}$ 's Using LLL: In the absence of round-offs, any $i$ th-frequency $(i \neq \mathrm{dc}) 3-\mathrm{D}$ DCT vector $X_{F, i}$ belongs to $\mathcal{L}_{i}$, which is spanned by a $\pi / 3$-orthogonal basis $\mathcal{T} \mathcal{Q}_{i}$. We seek to estimate a weakly $\pi / 3$-orthogonal basis $\mathcal{B}_{i}$ from all the $X_{F, i}$ 's. Given the $X_{F, i}$ 's $\in \mathcal{L}_{i}$ as inputs, LLL returns a reduced basis that spans all the $X_{F, i}$ 's. (The LLL-reduced basis is nonsingular when the input $X_{F, i}$ 's span $\mathcal{L}_{i}$; we will index these frequencies by $i \in\{1, \ldots, p\}$.) The LLL-reduced basis is not guaranteed to be weakly $\pi / 3$-orthogonal according to known worst-case bounds on LLL's performance. ${ }^{6}$ If the LLL-reduced basis is not $\pi / 3$-orthogonal, then we would need to search for a unimodular matrix $\mathcal{U}$ such that $\mathcal{B}_{i}$ is equal to an LLL-reduced basis times $\mathcal{U}$, and $\mathcal{B}_{i}$ is $\pi / 3$-orthogonal. However, in our experience, this search has been unnecessary. The LLL output has always been weakly $\pi / 3$-orthogonal for our lattices, which are "well-posed" in the sense that they contain at least one weakly $\pi / 3$-orthogonal basis. Our experience conforms with common knowledge that the LLL perform significantly better in practice than what is guaranteed theoretically [16], [17].

2) Estimating $\mathcal{T} \mathcal{Q}_{i}$ 's: Since $\mathcal{B}_{i}$ and $\mathcal{T} \mathcal{Q}_{i}$ are both bases for $\mathcal{L}_{i}$, we have

$$
\mathcal{B}_{i}=\mathcal{T} \mathcal{Q}_{i} \mathcal{U}_{i}
$$

for some unimodular matrix $\mathcal{U}_{i}$ (not necessarily the identity matrix). Hence, estimating the $\mathcal{T} \mathcal{Q}_{i}$ 's from the $\mathcal{B}_{i}$ 's is equivalent to decoding the respective $\mathcal{U}_{i}$ 's.

Thanks to the problem's structure, all the $\mathcal{U}_{i}$ 's satisfy the following constraints.

\footnotetext{
${ }^{4}$ Actually, some column permutation of $\mathcal{T}$ is weakly $(\pi / 3)+\epsilon$-orthogonal. For brevity, we do not emphasize this minor issue.

${ }^{5}$ Theoretically, our approach can be modified to accommodate cases where $\mathcal{T}$ is not weakly $(\pi / 3)+\epsilon$-orthogonal. However, as the $\mathcal{T}$ 's orthogonality decreases, that approach's computational demands would increase and its stability would deteriorate.

${ }^{6}$ For 2-D lattices, the LLL is guaranteed to return a weakly $\pi / 3$-orthogonal basis.
} 
1) $\mathcal{B}_{i} \mathcal{U}_{i}^{-1}$ is weakly $\pi / 3$-orthogonal. This follows from (22).

2) The columns of $\mathcal{U}_{i}$ corresponding to $\mathcal{B}_{i}$ 's shortest columns are the standard unit vectors times \pm 1 . This follows from Theorem 1 . Since both $\mathcal{B}_{i}$ and $\mathcal{T} \mathcal{Q}_{i}$ are weakly $\pi / 3$-orthogonal, they indeed contain the shortest vectors in the $\mathcal{L}_{i}$.

3) The product $\mathcal{U}_{i} \mathcal{B}_{i}^{-1} \mathcal{B}_{j} \mathcal{U}_{j}^{-1}$ is a positive diagonal matrix for any $i, j \in\{1, \ldots, p\}$. This follows from (22). Let $\widetilde{\mathcal{U}}_{i}$ 's, $i \in\{1, \ldots, p\}$ be arbitrary unimodular matrices that satisfy this property for every pair $i, j \in\{1, \ldots, p\}$. It is easy to show that if $\widetilde{\mathcal{U}}_{j} \neq \mathcal{U}_{j}$ for some $j$, then $\widetilde{\mathcal{U}}_{i} \neq \mathcal{U}_{i}$ for every $i \in\{1, \ldots, p\}$. It follows that to correctly estimate all the $\mathcal{U}_{i}$ 's, we just need to correctly estimate any one $\mathcal{U}_{i}$. Further, multiple unimodular sequences can satisfy this constraint only when the $\mathcal{Q}_{i}$ 's are chosen carefully. ${ }^{7}$

4) All nonzero elements of $\mathcal{U}_{i}$ are less than $\kappa\left(\mathcal{B}_{i}\right)$ in magnitude. This follows from Theorem 2.

Theoretically, the above conditions are not sufficient to uniquely determine the $\mathcal{U}_{i}$ 's. However, even with a small number $p$ of nonsingular $\mathcal{B}_{i}$ 's, the above conditions become so restrictive (particularly, constraint 3 ) that only the actual $\mathcal{U}_{i}$ 's satisfy all them simultaneously in practice. Hence, we just need to search for a unimodular matrix sequence that satisfy the above constraints.

The search focuses on estimating the correct unimodular matrix $\mathcal{U}_{\ell}$ for frequency $\ell:=\arg \min _{i \in\{1, \ldots, p\}} \kappa\left(\mathcal{B}_{i}\right)$. Let $\widetilde{\mathcal{U}}_{\ell}$ denote an arbitrary unimodular matrix that satisfies conditions 1,2 , and 4 for frequency $\ell$. From Theorem 2 , the frequency $\ell$ contains the least number of valid $\widetilde{\mathcal{U}}_{\ell}$ 's (along with the correct $\widetilde{\mathcal{U}}_{\ell}$ ), which makes the search for the correct $\mathcal{U}_{\ell}$ easier. We sequentially test each valid $\widetilde{\mathcal{U}}_{\ell}$ and verify if its choice allows us to find valid unimodular matrices $\widetilde{\mathcal{U}}_{i}$ 's for every frequency $i \in\{1, \ldots, p\}$. Note that given $\widetilde{\mathcal{U}}_{\ell}$, there exists at most one unimodular matrix $\widetilde{\mathcal{U}}_{i}$ such that $\widetilde{\mathcal{U}}_{\ell} \mathcal{B}_{\ell}^{-1} \mathcal{B}_{i} \widetilde{\mathcal{U}}_{i}^{-1}$ is positive diagonal; such a $\widetilde{\mathcal{U}}_{i}$ can be found easily, if it exists. Since the unimodular matrix conditions are extremely restrictive, in practice, we can safely assume that we will be able to find valid $\widetilde{\mathcal{U}}_{i}$ 's for all frequencies only if $\widetilde{\mathcal{U}}_{\ell}=\mathcal{U}_{\ell}$. Further, if $\widetilde{\mathcal{U}}_{i}=\mathcal{U}_{\ell}$, then $\widetilde{\mathcal{U}}_{i}=\mathcal{U}_{i}$. Thus, we can quickly and reliably determine the desired sequence of $\mathcal{U}_{i}$ 's from the $\mathcal{B}_{i}$ 's.

3) Decomposing $\mathcal{T} \mathcal{Q}_{i}$ into $\mathcal{T}$ and $\mathcal{Q}_{i}$ : Decomposing the $\mathcal{T} \mathcal{Q}_{i}$ 's into $\mathcal{T}$ and $\mathcal{Q}_{i}$ 's is equivalent (apart from the sign) to determining the norm of each column of $\mathcal{T}$ because the $\mathcal{Q}_{i}$ 's are diagonal matrices. The signs of $\mathcal{T}$ 's column are chosen such that its largest magnitude entry is positive. Let $\mathcal{T}(:, k)$ and $\left(\mathcal{T} \mathcal{Q}_{i}\right)(:, k), k=\{1,2,3\}$, denote $\mathcal{T}$ 's and $\mathcal{T} \mathcal{Q}_{i}$ 's $k$ th column vectors respectively. Then

$$
\left\|\left(\mathcal{T} \mathcal{Q}_{i}\right)(:, k)\right\|_{2}=q_{i, k}\|\mathcal{T}(:, k)\|_{2} .
$$

Since the $\mathcal{Q}_{i}$ 's diagonal elements $q_{i, k} \in \mathbb{Z}$, all elements of the set $\left\{\left\|\left(\mathcal{T} \mathcal{Q}_{i}\right)(:, k)\right\|_{2} \mid i \in\{1, \ldots, p\}\right\}$ lie on the same one-dimensional (1-D) lattice. The length of the shortest vector in this lattice is $\|\mathcal{T}(:, k)\|_{2}$. Hence, we set $\mathcal{T}$ 's column norm to be the length of the shortest nonzero vector in the 1-D lattice comprising the $\left\|\left(\mathcal{T} \mathcal{Q}_{i}\right)(:, k)\right\|_{2}$ 's.

\footnotetext{
${ }^{7}$ For example, this is possible if all the $\mathcal{Q}_{i}$ 's are $\mathcal{Q}_{1}$ 's scaled versions and the
} actual $\mathcal{U}_{i}$ 's are all identity matrices. Then, any sequence $\left\{\widetilde{\mathcal{U}}_{i}\right\}$ with $\widetilde{\mathcal{U}}_{i}=\widetilde{\mathcal{U}}_{1}$, $i \in\{2, \ldots, p\}$ satisfies constraint 3 . Note that to make $\left\{\tilde{\mathcal{U}}_{i}\right\}$ simultaneously satisfy the other three constraints, $\mathcal{Q}_{1}$ must be chosen carefully.
4) Estimating the DC Quantization Step-Sizes and the Color Transform's Additive Component: The dc coefficients in the observed space, after being transformed by the estimated $\mathcal{T}$ 's inverse, is related to compression color space dc coefficients by [see (3)]

$$
\mathcal{T}^{-1} X_{\mathrm{F}, \mathrm{dc}}=\bar{X}_{\mathrm{G}, \mathrm{dc}}-\mathrm{DCT} \text { of additive component. }
$$

Since $\bar{X}_{\mathrm{G}, \mathrm{dc}}$ lies on a rectangular box lattice, $\mathcal{T}^{-1} X_{\mathrm{F}, \mathrm{dc}}$ lies on a shifted rectangular box lattice. The dc quantization stepsize can be obtained by first subtracting an arbitrary reference vector $\mathcal{T}^{-1} X_{\mathrm{F}, \mathrm{dc}}$ from all the vectors to nullify the shift and then solving the SVP problem along each component.

We estimate the additive component by exploiting two constraints. First, the additive component should be such that the $\bar{X}_{\mathrm{G}, \mathrm{dc}}$ [see (24)] lie on a lattice. Hence, at least some $\bar{X}_{\mathrm{G}, \mathrm{dc}}$ should be zero. Second, all

$$
X_{\mathrm{F}, \mathrm{dc}}+\mathcal{T} \times \text { DCT of additive component }
$$

should lie in the 0-255 range after transformation. Many additive component estimates could satisfy the above two criteria. In such cases, we arbitrarily pick one of the solutions. Note that errors in the additive component estimates do not significantly affect applications such as recompression and enhancement since these applications merely shift the dc coefficients in the compression color space.

\section{B. Robustification to Round-Off Noise}

We now clarify the modifications required to combat round-off noise.

1) Estimating $\pi / 3$-Orthogonal Bases $\mathcal{B}_{i}$ 's Using a Robustified LLL Algorithm: We desire to estimate a nearly orthogonal basis $\widehat{\mathcal{B}}_{i}$ such that all the 3-D DCT vectors $\widetilde{X}_{F, i}$ 's lie close to the lattice spanned by $\widehat{\mathcal{B}}_{i}$ [see (18)]. The conventional LLL is unstable when the input vectors $\widetilde{X}_{F, i}$ 's contain noise. Hence to estimate $\widehat{\mathcal{B}}_{i}$, we stabilize LLL in two ways.

First, we exploit the multiplicity of noisy lattice vector realizations to reduce round-off noise propagation. We observe multiple noisy realizations of the lattice vectors because even reasonable-sized images contain many $8 \times 8$ pixel blocks. We input the least noisy vectors first to LLL. To determine this input order, we compute the histograms of all the $i$ th-frequency 3-D DCT vectors, and sort the vectors in the descending order of histogram values.

Second, we incorporate a least-squares noise attenuation step that exploits the round-off errors' statistical distribution. Given a lattice basis estimate $\widetilde{\mathcal{B}}_{i}$, we assume that any vector lying within a distance $\zeta$ (chosen adaptively from the range $[3.5,5]$ ) from its closest point on the lattice spanned by $\widetilde{\mathcal{B}}_{i}$ is just a noisy realization. We use the noisy realization to update the $\widetilde{\mathcal{B}}_{i}$ and obtain $\widehat{\mathcal{B}}_{i}$. Let $\mathcal{D}_{i}$ denote a $3 \times m$ matrix containing the $\widetilde{X}_{F, i}$ 's as its columns. Let $\mathcal{I}_{i} \in \mathbb{Z}^{3 \times m}$ be such that each column of $\widetilde{\mathcal{B}}_{i} \mathcal{I}_{i}$ is the closest point on the lattice spanned by $\widetilde{\mathcal{B}}_{i}$ to the corresponding column of $\mathcal{D}_{i}$; that is

$$
\widetilde{\mathcal{B}}_{i} \widetilde{\mathcal{I}}_{i}=\operatorname{CVP}_{\widetilde{\mathcal{B}}_{i}}\left(\mathcal{D}_{i}\right), \quad \widetilde{\mathcal{I}}_{i} \in \mathbb{Z}^{3 \times m} .
$$

Assuming that the distance from $\mathcal{D}_{i}$ to the closest lattice point is less than $\zeta$, we have using (19) that

$$
P\left(\mathcal{D}_{i} \mid \widetilde{\mathcal{B}}_{i}\right) \propto \exp \left(-\frac{1}{2 \sigma^{2}}\left\|\mathcal{D}_{i}-\widetilde{\mathcal{B}}_{i} \widetilde{\mathcal{I}}_{i}\right\|_{H S}^{2}\right)
$$


where $\|.\|_{H S}^{2}$ denotes the squared Hilbert-Schmidt norm (the square root of the sum of all the matrix elements' squares). We can update the basis estimate as

$$
\widehat{\mathcal{B}}_{i}:=\arg \min _{\widetilde{\mathcal{B}}_{i}}\left\|\mathcal{D}_{i}-\widetilde{\mathcal{B}}_{i} \widetilde{\mathcal{I}}_{i}\right\|_{H S}^{2}=\left(\mathcal{D}_{i} \widetilde{\mathcal{I}}_{i}^{T}\right)\left(\widetilde{\mathcal{I}}_{i} \widetilde{\mathcal{I}}_{i}^{T}\right)^{-1} .
$$

Equation (27) assumes that the round-off error norms stay less than $\zeta$ and, hence, ignores the distribution's finite support. The estimation (27) naturally leads to an iterative update where $\mathcal{I}$ is recomputed using (25) with $\widetilde{\mathcal{B}}_{i}=\widehat{\mathcal{B}}_{i}$. As desired, this iteration is guaranteed to converge to a locally optimal $\widehat{\mathcal{B}}_{i}$ that minimizes the round-off error between the observations $\mathcal{D}_{i}$ and the closest points on the lattice spanned by $\widehat{\mathcal{B}}_{i} \cdot{ }^{8}$

In summary, we fuse LLL with noise attenuation as follows.

1) Compute the histogram of the ac frequency vectors and sort them in descending order of histogram values.

2) Include the first vector outside the radius $\zeta$ as a lattice basis vector. For each frequency $i$, the $\zeta \in[3.5,5]$ is set adaptively. Any vector within the sphere could potentially be a noisy realization of the origin $[0,0,0]^{T}$, and hence should be ignored.

3) Compute the error vector between the next ac frequency vector and the closest vector on the lattice (obtained by solving a CVP) spanned by the current basis estimate. If the error vector's norm is greater than $\zeta$, then include the currently chosen vector in the list of basis vectors, and perform LLL on this set of basis vectors. If the error vector norm is less or equal to $\zeta$, then update the basis vectors using (27).

Combining the update step with LLL successfully curbs the propagation and amplification of the round-off errors during LLL's arithmetic operations.

2) Estimating the $\mathcal{T} \mathcal{Q}_{i}$ 's: In the absence of round-offs, there exist $\mathcal{U}_{i}$ 's such that $\mathcal{U}_{i} \mathcal{B}_{i}^{-1} \mathcal{B}_{j} \mathcal{U}_{j}^{-1}$ is exactly diagonal (see Section VIII-A2). However, due to round-offs, $\mathcal{U}_{i} \widehat{\mathcal{B}}_{i}^{-1} \widehat{\mathcal{B}}_{j} \mathcal{U}_{j}^{-1}$ can only be diagonally dominant. We define the diagonality of a matrix as the $\ell_{2}$ norm of the matrix's diagonal elements divided by the $\ell_{2}$ norm of all the matrix elements; the measure is equal to one if and only if the matrix is exactly diagonal. We estimate unimodular matrices $\widehat{\mathcal{U}}_{i}$ 's such that sum of the $\widehat{\mathcal{U}}_{i} \widehat{\mathcal{B}}_{i}^{-1} \widehat{\mathcal{B}}_{j} \widehat{\mathcal{U}}_{j}^{-1}$ 's diagonality measures is maximized. We set $\widehat{\mathcal{T}} \widehat{\mathcal{Q}}_{i}:=\widehat{\mathcal{B}}_{i} \widehat{\mathcal{U}}_{i}^{-1}$, with $\widehat{\mathcal{T}}$ and $\widehat{\mathcal{Q}}_{i}$ denoting $\mathcal{T}$ 's and $\mathcal{Q}_{i}$ 's estimates respectively.

3) Estimating $\mathcal{T}$ and $\mathcal{Q}_{i}$ 's: The estimated $\widehat{\mathcal{T}} \widehat{\mathcal{Q}} ;$ 's column norms conform only approximately to a 1-D lattice spanned by the corresponding column norm of the true $\mathcal{T}$. Similar to the quantization step-size estimation described in Section IV, we estimate $\hat{\mathcal{T}}$ 's column norms by solving a penalized least-squares cost function, as shown by (28) at the bottom of the page)

\footnotetext{
${ }^{8}$ Convergence follows because both (25) and (27) monotonically reduce $\left\|\mathcal{D}_{i}-\widetilde{\mathcal{B}}_{i} \mathcal{I}_{i}\right\|_{H S}^{2}$.
}

The first term ensures that $\widehat{\mathcal{T}} \widehat{\mathcal{Q}}_{i}$ 's column norms conform to a 1 -D lattice spanned by $\varrho$, and the second term ensures that $\widehat{\mathcal{T}}$ 's column norm is large. The $\beta$ controls the tradeoff between the two terms. In practice, we set $\beta:=0.2 / \operatorname{mean}\left(\left\|\left(\mathcal{T} \mathcal{Q}_{i}\right)(:, k)\right\|_{2}\right)$. We can then estimate the quantization step-sizes for all the ac frequencies as

$$
\widehat{q}_{i, k}=\operatorname{round}\left(\frac{\left\|\left(\widehat{\mathcal{T}} \widehat{\mathcal{Q}}_{i}\right)(:, k)\right\|_{2}}{\|\widehat{\mathcal{T}}(:, k)\|_{2}}\right) \text {. }
$$

4) Estimating the Additive Component and the DC Quantization Step-Sizes: Each entry of an arbitrary vector $\widehat{\mathcal{T}}^{-1} \widetilde{X}_{\mathrm{F}, \mathrm{dc}}$ is approximately equal to an integer multiple of the respective dc quantization step-size plus a constant shift. Hence, the histograms of the collection of $\widehat{\mathcal{T}}^{-1} \widetilde{X}_{\mathrm{F}, \mathrm{dc}}$ 's respective entries look like shifted versions of Fig. 3. We note that the histogram's discrete Fourier transform (DFT) magnitude is immune to the unknown constant shift. Further, the DFT's peak frequency captures the histogram's "periodicity," which is determined by the quantization step-size. Hence, we estimate the quantization step-size as the inverse of the nonzero frequency at which the histogram's DFT magnitude peaks. Subsequently, we estimate the affine transform's additive component as described in Section VIII-A.

\section{Lattice-Based CHEst Results}

We demonstrate the performance on lattice-based CHEst on the $512 \times 512$ Lena color image [12] that we JPEG-compressed in the ITU.BT-601 YCbCr space [see (3)]. Lattice-based CHEst performed equally well on a wide variety of other experiments comprising different images and compression color spaces. The luminance plane $Y$ 's DCT coefficients were quantized using table 1 from Fig. 2 and the chrominance planes $C b$ 's and $C r$ 's DCT coefficients were quantized using table 2 from Fig. 2 . The $\mathrm{Cb}$ and $\mathrm{Cr}$ planes were not subsampled during compression. The image was then decompressed and then transformed to the $R G B$ space. The algorithm operated in this $R G B$ space and tried to estimate the affine transformation from ITU.BT-601 $Y C b C r$ to the current $R G B$ space [see (3)].

Lattice-based CHEst estimated that the affine transform from the compression space ITU.BT-601 YCbCr to the observation space $R G B$ was

$$
\left[\begin{array}{l}
R \\
G \\
B
\end{array}\right]=\left[\begin{array}{ccc}
1.00 & 0.00 & 1.41 \\
1.00 & -0.35 & -0.71 \\
1.00 & 1.78 & 0.00
\end{array}\right]\left(\left[\begin{array}{c}
Y \\
C b \\
C r
\end{array}\right]-\left[\begin{array}{c}
3 \\
88 \\
138
\end{array}\right]\right)
$$

Fig. 6 illustrates the algorithm's quantization table estimates. An $\times$ indicates that the quantization step-size estimation was not possible because all DCT coefficients were quantized to zero. The estimated $\mathrm{CH}$ conforms well with the true compression settings; compare (3) to (29) and Fig. 2 to Fig. 6.

$$
\|\widehat{\mathcal{T}}(:, k)\|=\arg \min _{\varrho} \sum_{i}\left(\left(\left\|\left(\widehat{\mathcal{T}} \widehat{\mathcal{Q}}_{i}\right)(:, k)\right\|-\varrho \text { round }\left(\frac{\left\|\left(\widehat{\mathcal{T}} \widehat{\mathcal{Q}}_{i}\right)(:, k)\right\|}{\varrho}\right)\right)^{2}+\beta \text { round }\left(\frac{\left\|\left(\widehat{\mathcal{T}} \widehat{\mathcal{Q}}_{i}\right)(:, k)\right\|}{\varrho}\right)\right) .
$$



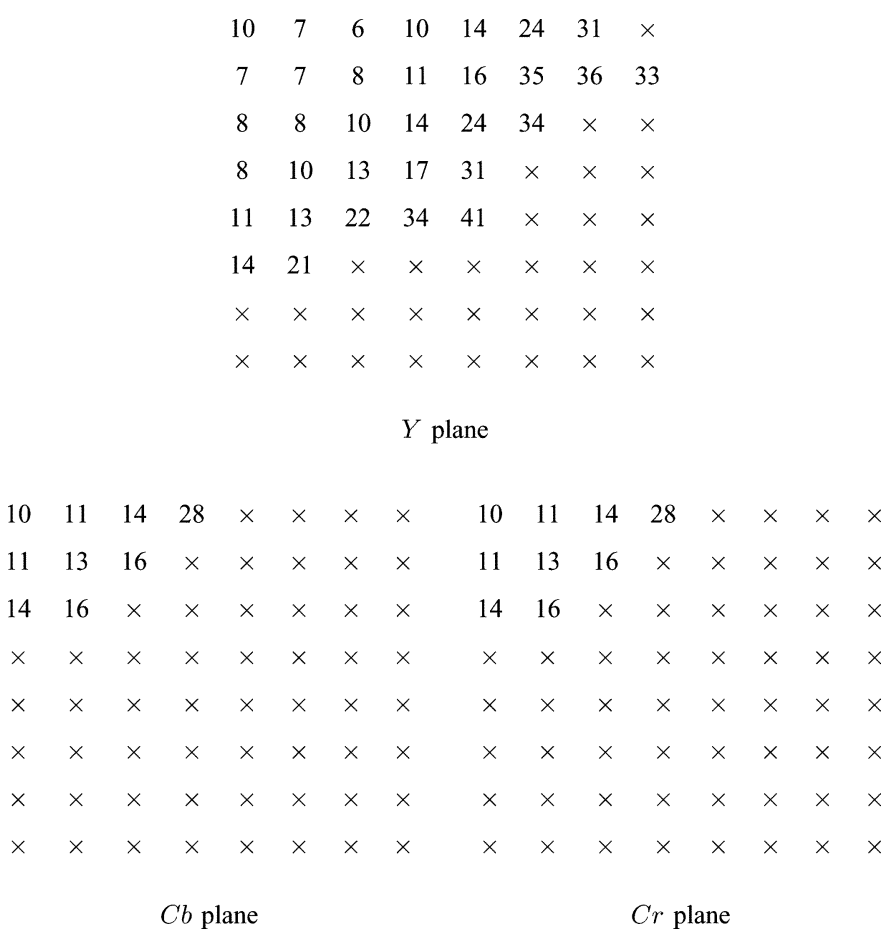

Fig. 6. Quantization table estimates using lattice-based CHEst.

We now outline the results obtained by the algorithm's various intermediate steps. In the first step (see Step 1 in Section VIII-A), robustified LLL (details in Section VIII-B1) estimated the lattice bases for the ac frequencies $(1,2)$ and $(1,3)$, as

$$
\begin{aligned}
& \widehat{\mathcal{B}}_{(1,2)}=\left[\begin{array}{ccc}
-7.00 & 15.50 & -6.96 \\
-7.01 & -7.81 & -10.72 \\
-7.00 & 0.02 & 12.66
\end{array}\right] \text { and } \\
& \widehat{\mathcal{B}}_{(1,3)}=\left[\begin{array}{ccc}
-6.01 & 13.64 & -5.94 \\
-5.99 & -15.91 & -10.88 \\
-6.00 & -5.91 & 18.95
\end{array}\right] .
\end{aligned}
$$

Clearly, the respective first columns of $\widehat{\mathcal{B}}_{(1,2)}$ and $\widehat{\mathcal{B}}_{(1,3)}$, which are the shortest columns, are indeed aligned with one of the columns of the ITU.BT-601 $\mathrm{YCbCr}$ to $\mathrm{RGB}$ transformation's linear component $\mathcal{T}$. However, $\widehat{\mathcal{B}}_{(1,2)}$ 's third column and $\widehat{\mathcal{B}}_{(1,2)}$ 's second and third column are not scaled versions of any of $\mathcal{T}$ 's columns due to the addition of the first column.

In the second step (see Step 2 in Section VIII-A), we deduced the unimodular matrices to be

$\widehat{\mathcal{U}}_{(1,2)}=\left[\begin{array}{ccc}-1 & 0 & -1 \\ 0 & 1 & 0 \\ 0 & 0 & 1\end{array}\right]$ and $\widehat{\mathcal{U}}_{(1,3)}=\left[\begin{array}{ccc}-1 & -1 & -1 \\ 0 & 1 & 0 \\ 0 & 0 & 1\end{array}\right]$.

Hence, the estimate $\widehat{\mathcal{T}} \widehat{\mathcal{Q}}_{i}=\widehat{\mathcal{B}}_{i} \widehat{\mathcal{U}}_{i}^{-1}$ for the ac frequencies $(1,2)$ and $(1,3)$ is

$$
\begin{aligned}
& \widehat{\mathcal{B}}_{(1,2)} \widehat{\mathcal{U}}_{(1,2)}^{-1}=\left[\begin{array}{ccc}
7.00 & 15.50 & 0.04 \\
7.01 & -7.81 & -3.71 \\
7.00 & 0.02 & 19.66
\end{array}\right] \text { and } \\
& \widehat{\mathcal{B}}_{(1,3)} \widehat{\mathcal{U}}_{(1,3)}^{-1}=\left[\begin{array}{ccc}
6.01 & 19.65 & 0.07 \\
5.99 & -9.92 & -4.89 \\
6.00 & 0.09 & 24.95
\end{array}\right] .
\end{aligned}
$$

Similarly, we computed the $\widehat{\mathcal{T}} \widehat{\mathcal{Q}}_{i}$ for all the ac frequencies.
In the third step (see Step 3 in Section VIII-A), we estimated $\mathcal{T}$ 's column norms using (28). This yielded all the ac frequency quantization step-sizes $\widehat{\mathcal{Q}}_{i}$ illustrated in Fig. 6 and the $\mathcal{T}$ (the $3 \times 3$ matrix) in (29).

In the fourth and final step (see Step 4 in Section VIII-A), we used the dc frequency coefficients to estimate the additive component as the $3 \times 1$ matrix in (29), and the dc quantization step-sizes as shown in Fig. 6.

\section{JPEG RECOMPRESSION: An EXAMPle APPliCATION OF CHEst}

When a given TIFF or BMP image's file-size needs to be reduced, the conventional approach is to naively employ JPEG with an arbitrary choice of compression color space, subsampling factor, and quantization table. For naive JPEG recompression, reasonable choices for the color transformations include RGB to ITU.BT-601 YCbCr, Computer RGB to ITU.BT-601 $Y C b C r, R G B$ to Kodak PhotoYCC, and $s R G B$ to 8-bit CIELab. Some common subsampling factors are $2 \times 2,1 \times 1,1 \times 1$ and $1 \times 1,1 \times 1,1 \times 1$. The quantization tables are often set by adjusting a so-called quality factor $(\mathrm{QF})$, a reference number between 1 to 100 used by the IJG JPEG implementation [9]; $\mathrm{QF}=100$ sets all the quantizer step-sizes to unity and thus yields the best quality JPEG can possibly achieve. Any combination of the above choices would yield a JPEG image file with a certain file-size. Smaller file-sizes are typically accompanied by increased distortions in the recompressed image. In this section, we demonstrate that using CHEst to recompress a JPEG-decompressed color image offers significant benefits over a naive recompression approach.

\section{A. JPEG Recompression Using Dictionary-Based CHEst}

To demonstrate the dictionary-based CHEst's benefits in JPEG recompression, consider the same test image described in Section V-C-Lena color image previously JPEG-compressed in the 8-bit CIELab color space with $2 \times 2,1 \times 1,1 \times 1$ subsampling using quantization table 1 and 2 from Fig. 2. As described in Section V-C, dictionary-based CHEst accurately estimates the test image's $\mathrm{CH}$.

To perform recompression using the dictionary-based CHEst information, we first transformed the observed image into the estimated compression color space using the $s R G B$ to 8-bit CIELab color transformation. Then, we deconvolved the effect of the smoothing employed during previous decompression on the $a$ and $b$ color planes. After performing $2 \times 2,1 \times 1$, $1 \times 1$ subsampling, using the IJG JPEG implementation [9], we JPEG-compressed the 8-bit CIELab color planes with the estimated quantization tables in Fig. 4 (setting the $\times$ entries to 100). Our recompression yielded a JPEG image with file-size 32.31 kilobytes $(\mathrm{kB})$ with an SNR of $22.58 \mathrm{~dB}$. The SNR is computed with respect to the original Lena image in the perceptually-uniform CIELab color space. We also visually inspected the images to confirm that the SNR values were qualitatively consistent with the image's perceptual quality.

For comparison, we also recompressed the image using a variety of naively chosen settings. We JPEG-compressed the test BMP image using the $R G B$ to ITU.BT-601 YCbCr, Computer $R G B$ to ITU.BT-601 YCbCr, RGB to Kodak PhotoYCC, and 


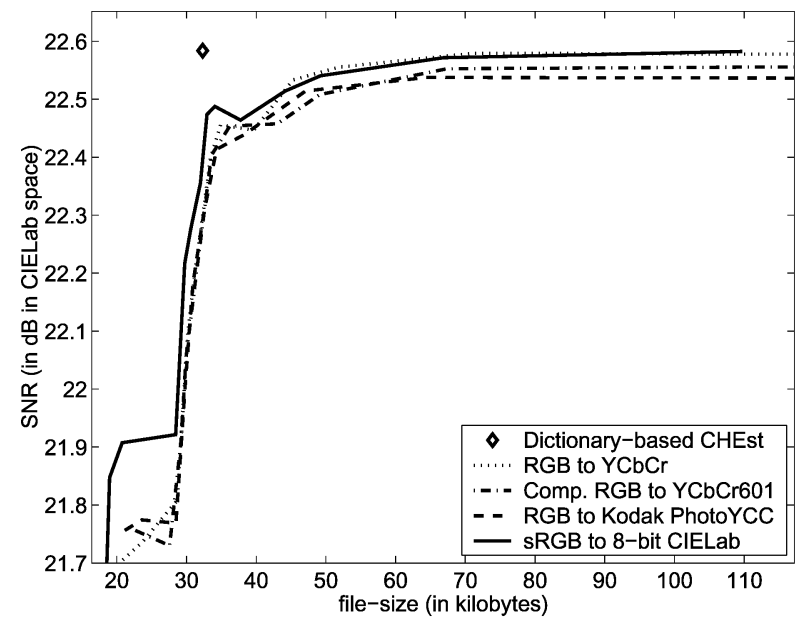

(a) $2 \times 2,1 \times 1,1 \times 1$ subsampling

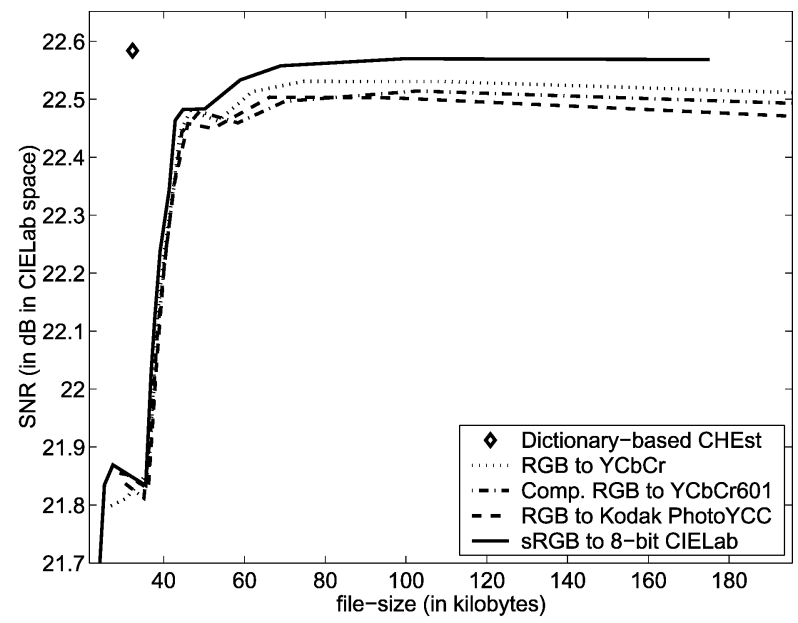

(b) $1 \times 1,1 \times 1,1 \times 1$ subsampling

Fig. 7. JPEG recompression results for dictionary-based CHEst. The " $\diamond$ " marks the file-size SNR pair $(32.31 \mathrm{kB}, 22.58 \mathrm{~dB})$ obtained using dictionary-based CHEst results for JPEG recompression. Each curve in (a) illustrates the achieved file-size versus SNR tradeoff for naive recompression in the indicated color space with $2 \times 2,1 \times 1,1 \times 1$ subsampling. Plot (b) illustrates the tradeoff when $1 \times 1,1 \times 1,1 \times 1$ subsampling is employed.

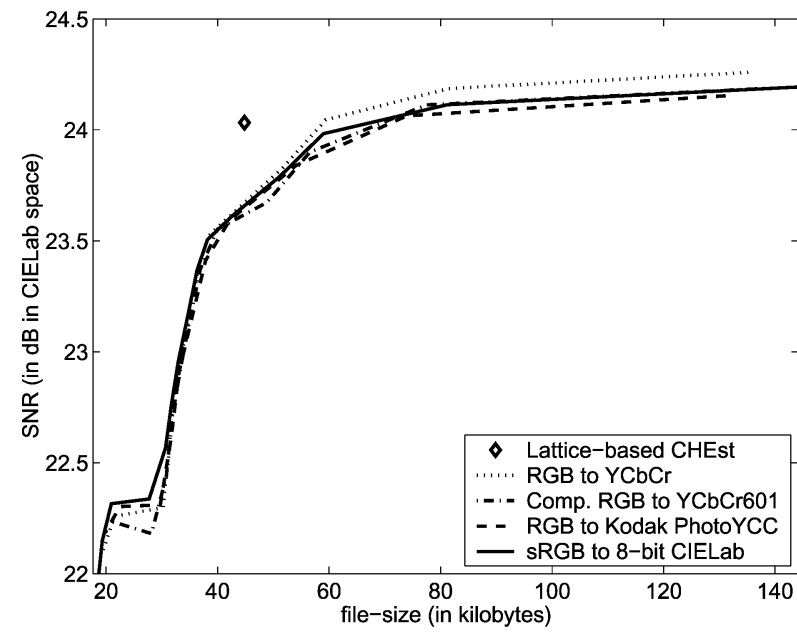

(a) $2 \times 2,1 \times 1,1 \times 1$ subsampling

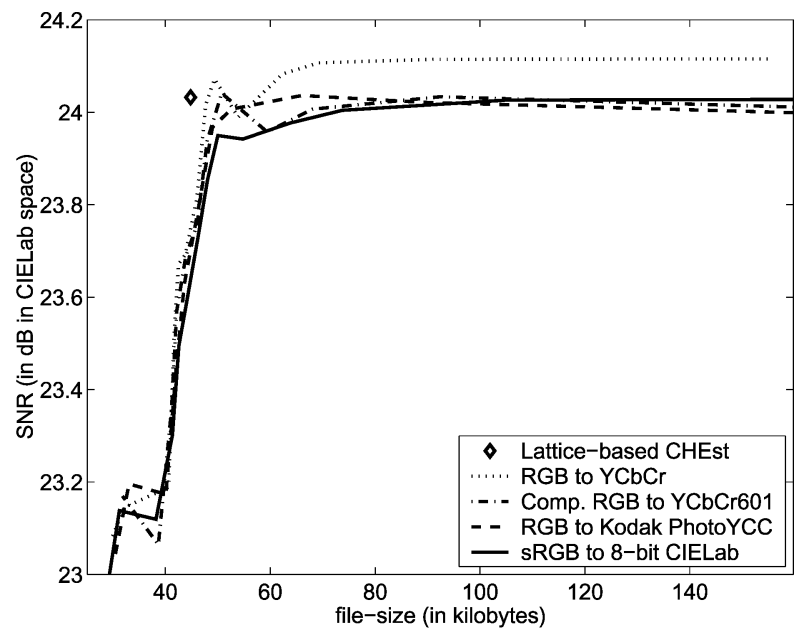

(b) $1 \times 1,1 \times 1,1 \times 1$ subsampling

Fig. 8. Recompression results for lattice-based CHEst. Recompression using lattice-based CHEst information yields a JPEG image whose file-size is $44.81 \mathrm{kB}$ and SNR is $24.03 \mathrm{~dB}$; a " $\diamond$ " marks this file-size SNR pair. Similar to Fig. 7, the curves (a) and (b) illustrate the achieved file-size versus SNR tradeoff for naive recompression in the indicated color space with $2 \times 2,1 \times 1,1 \times 1$ and $1 \times 1,1 \times 1,1 \times 1$ subsampling, respectively.

$s R G B$ to 8-bit CIELab color transforms using $2 \times 2,1 \times 1$, $1 \times 1$, and also using $1 \times 1,1 \times 1,1 \times 1$ subsampling. For each chosen color transform and subsampling, we varied the quantization tables using the $\mathrm{QF}$ value and noted the resulting JPEG image's file-size (in kilobytes) and the incurred distortion in SNR (in decibels in the CIELab space).

Figs. 7(a) and (b) summarize the recompression results. In both plots, the " $\diamond$ " symbol marks the file-size SNR pair (32.31 kB, $22.58 \mathrm{~dB}$ ) associated with the image recompressed using dictionary-based CHEst results. Each curve in Fig. 7(a) illustrates the achieved file-size versus SNR tradeoff for naive recompression in the indicated color space with $2 \times 2,1 \times 1,1 \times 1$ subsampling. The curves in Fig. 7(b) illustrate the tradeoff when $1 \times 1$, $1 \times 1,1 \times 1$ subsampling is employed. The naive recompression curves demonstrate a "knee-point" trend-the SNR remains flat for a broad file-size range, but decreases rapidly for small file-size changes thereafter. (Arguments similar to those in [4] could be used to explain the nonmonotonicity of the naive recompression curves.) Both the plots confirm that exploiting the dictionarybased CHEst enables us to strike a desirable file-size versus distortion tradeoff-we attain the nearly minimum file-size without introducing significant additional distortion.

\section{B. JPEG Recompression Using Lattice-Based CHEst}

We demonstrate the lattice-based CHEst's benefits in JPEG recompression using the test image described in Section VIII-C-Lena color image previously JPEG-compressed in the ITU.BT-601 YCbCr color space with $1 \times 1,1 \times 1,1 \times$ 1 subsampling using quantization tables 1 and 2 from Fig. 2 . As described in Section VIII-C, lattice-based CHEst accurately estimates the test image's $\mathrm{CH}$.

To perform recompression using the lattice-based CHEst results, we transformed the observed image to the estimated compression space using the inverse of estimated ITU.BT-601 $\mathrm{YCbCr}$ to $\mathrm{RGB}$ transformation in (29). We JPEG-compressed the three planes using the estimated quantization tables in Fig. 2 
(setting the $\times$ entries to 100) to obtain an image with file-size $=44.81 \mathrm{kB}$ and $\mathrm{SNR}=24.03 \mathrm{~dB}$.

Figs. 8(a) and (b) compare the file-size SNR pair for latticebased CHEst recompression with file-size versus SNR curves for naive JPEG recompression in different color spaces at different QFs for $2 \times 2,1 \times 1,1 \times 1$ and $1 \times 1,1 \times 1,1 \times$ 1 subsampling. Fig. 8 verifies that lattice-based CHEst results also enables us to strike a desirable file-size versus distortion tradeoff during JPEG recompression.

\section{CONCLUSION}

This paper has addressed the JPEG CHEst problem for color images and its potential applications. JPEG compression leaves its signature on an image by quantizing the image's DCT coefficients and forcing them to closely conform to near-periodic structures. We have described two new approaches that exploit these structures to solve the CHEst problem.

First, we formulated a statistical framework to characterize and exploit the JPEG-induced near-periodic structures for gray-scale and color images. Essentially, the statistical approach chooses from a dictionary the best $\mathrm{CH}$ model that explains the regular structure of the observed image's DCT coefficients.

Second, for cases when JPEG employs affine color transforms and no subsampling, we devised a blind CHEst scheme that does not rely on a finite dictionary. In this case, the JPEGdecompressed image's DCT coefficients conform to 3-D lattice structures. The JPEG CH information is encoded in the nearly orthogonal bases that span the DCT lattices. By exploiting recent insights on nearly orthogonal lattice bases and existing lattice algorithms, we provided a novel blind lattice-based solution to the CHEst problem.

JPEG CHEst offers significant benefits during the recompression of JPEG-decompressed color images compared to a naive approach. We demonstrated that using the estimated $\mathrm{CH}$ during JPEG recompression introduces minimal distortion (large SNR) and simultaneously achieves a small file-size.

JPEG CHEst could also help us uncover proprietary compression settings used by imaging devices. It could contribute to applications such as covert message passing and image authentication. In summary, we envision that JPEG CHEst would enable a variety of intriguing applications.

\section{ACKNOWLEDGMENT}

The authors would like to thank M. Gaubatz for the color transform implementation and the reviewers for their insightful suggestions.

\section{REFERENCES}

[1] C. Poynton, A Technical Introduction to Digital Video. New York: Wiley, 1996.

[2] G. Sharma and H. Trussell, "Digital color imaging," IEEE Trans. Image Process., vol. 6, no. 6, pp. 901-932, Jul. 1997.

[3] W. Pennebaker and J. Mitchell, JPEG, Still Image Data Compression Standard. New York: Van Nostrand, 1993.

[4] H. H. Bauschke, C. H. Hamilton, M. S. Macklem, J. S. McMichael, and N. R. Swart, "Recompression of JPEG images by requantization," IEEE Trans. Image Process., vol. 12, no. 6, pp. 843-849, Jul. 2003.
[5] Z. Fan and R. Eschbach, "JPEG decompression with reduced artifacts," in Proc. IS\&T/SPIE Symp. Electronic Imaging: Image and Video Compression, San Jose, CA, Feb. 1994, pp. 50-55.

[6] Z. Fan and R. de Queiroz, "Identification of bitmap compression history: JPEG detection and quantizer estimation," IEEE Trans. Image Process., vol. 12, no. 2, pp. 230-235, Feb. 2003.

[7] R. Neelamani, "Inverse Problems in Image Processing," Ph.D. dissertation, Elec. Conput. Eng. Dept., Rice University, Houston, TX, 2003.

[8] R. Neelamani, S. Dash, and R. Baraniuk, "On nearly orthogonal lattice bases,” IBM, IBM Research Rep. RC 23620, Jun. 2005. Submitted to SIAM J. Discrete Math.: www.dsp.rice.edu/ neelsh/publications.

[9] Independent JPEG Group Library. IJG. [Online] Available: www.ijg.org

[10] R. J. Clarke, Transform Coding of Images. London, U.K.: Academic, 1985.

[11] A. N. Tikhonov and V. Y. Arsenin, Solutions of Ill-Posed Problems. Washington, DC: Winston, 1977.

[12] The USC-SIPI Image Database. [Online] Available: sipi.usc.edu/services/database/Database.html

[13] A. K. Lenstra, H. W. Lenstra Jr., and L. Lovász, "Factoring polynomials with rational coefficients," Math. Annalen, vol. 261, pp. 515-534, 1982.

[14] E. Agrell, T. Eriksson, A. Vardy, and K. Zeger, "Closest point search in lattices," IEEE Trans. Inf. Theory, vol. 48, no. 8, pp. 2201-2214, Aug. 2002.

[15] R. Kannan, "Algorithmic geometry of numbers," in Annu. Rev. Comput. Sci., vol. 2, 1987, pp. 231-267.

[16] P. Nguyen and J. Stern, "Lattice reduction in cryptology: an update," in Lecture Notes in Comp. Sci.. New York: Springer Verlag, 2000, vol. 1838, pp. 85-112.

[17] A. Joux and J. Stern, "Lattice reduction: a toolbox for the cryptanalyst," J. Cryptol., vol. 11, no. 3, pp. 161-185, 1998

[18] G. H. Golub and C. F. V. Loan, Matrix Computations. Baltimore, MD: Johns Hopkins Univ. Press, 1989.

[19] M. Ajtai, "The shortest vector problem in $L_{2}$ is NP-hard for randomized reductions," in 30th Annu. ACM Symp. Theory Comput., 1998, pp. $10-19$.

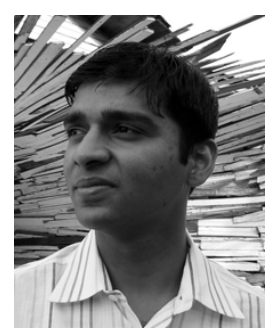

Ramesh (Neelsh) Neelamani (M'03) received the B.Tech. degree from the Indian Institute of Technology, Bombay, India, in 1997 and the M.S. and $\mathrm{Ph} . \mathrm{D}$. degrees from Rice University, Houston, TX, in 1999 and 2003, respectively, all in electrical engineering.

He is currently a Research Scientist at ExxonMobil Upstream Research Company, Houston. His research passions include signal and image processing topics such as multiscale signal representations and algorithms, statistical signal processing, seismic imaging, color processing, distributed algorithms, and pattern recognition.

Dr. Neelamani won the second prize in the IEEE all-India student paper contest in 1995 . He is a member of the SEG.

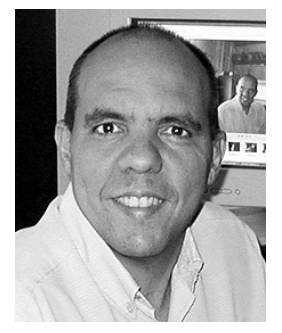

Ricardo de Queiroz (SM'99) received the Engineer degree from Universidade de Brasilia, Brasilia, Brazil, in 1987, the M.Sc. degree from the Universidade Estadual de Campinas, Campinas, Brazil, in 1990, and the Ph.D. degree from University of Texas, Arlington, in 1994, all in electrical engineering.

From 1990-1991, he was with the DSP research group at the Universidade de Brasilia as a Research Associate. He joined the the Xerox Corporation in 1994, where he was a member of the research staff until 2002. From 2000-2001, he was also an Adjunct Faculty at the Rochester Institute of Technology, Rochester, NY. He is now with the Electrical Engineering Department at Universidade de Brasilia. He has published extensively in journals and conferences and contributed chapters to books as well. He also holds 36 issued patents. His research interests include multirate signal processing, image and signal compression, and color imaging.

Dr. de Queiroz is a member of the IEEE Signal Processing Society Image and Multiple Dimensional Signal Processing (IMDSP) Technical Committee. He is an Associate Editor for the IEEE TRANSACTIONS ON IMAGE PROCESSING, IEEE TRANSACTIONS ON CIRCUITS AND SYSTEMS FOR VIDEO TECHNOLOGY and a past Editor for IEEE Signal PROCESSING LETTERS. He has been actively involved with the Rochester chapter of the IEEE Signal Processing Society, where he served as Chair and organized the Western New York Image Processing Workshop since its inception until 2001. He was also part of the organizing committee of ICIP'2002. 


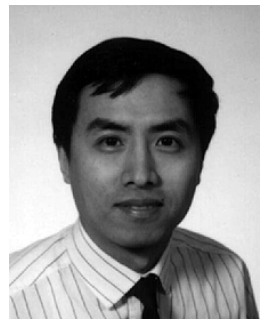

Zhigang Fan (SM'03) received the M.S. and Ph.D. degrees in electrical engineering from the University of Rhode Island, Kingston, in 1986 and 1988, respectively.

He joined the Xerox Corporation, Webster, NY, in 1988, where he is currently working as a Principal Scientist in Xerox Corporate Research and Technology. His research interests include various aspects of image processing and recognition, in particular color imaging, document image segmentation and analysis, and anticounterfeit and security printing. He has authored and coauthored more than 60 technical papers, as well as over 100 patents and pending applications.

Dr. Fan is an Associate Editor for the IEEE Transactions on IMAGE PROCESSING.

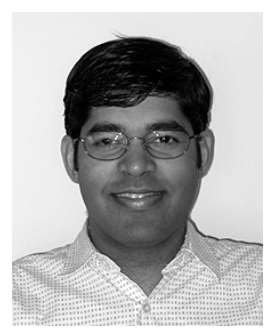

Sanjeeb Dash received the Ph.D. degree from Rice University, Houston, TX.

He is a Research Staff Member in the Mathematical Sciences Department of IBM's T. J. Watson Research Center, Yorktown Heights, NY, which he first joined as a Herman Goldstine Postdoctoral Fellow in 2002. He joined IBM after the Ph.D. degree and a stint as a Postdoctoral Fellow in the Program in Applied Mathematics at Princeton University, Princeton, NJ. His main research focus is in the areas of integer and linear programming, especially their computational aspects.

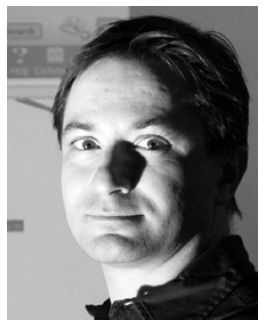

Richard G. Baraniuk (F'02) received the B.Sc. degree from the University of Manitoba, Winnipeg, in 1987, the M.Sc. degree from the University of Wisconsin, Madison, in 1988, and the Ph.D. degree from the University of Illinois, Urbana-Champaign, in 1992, all in electrical engineering.

After spending 1992-1993 at L'Ecole Normale Supérieure, Lyon, France, he joined Rice University, Houston, TX, where he is currently the Victor E. Cameron Professor of Electrical and Computer Engineerng and Director of the Connexions Project. He spent sabbaticals at L'Ecole Nationale Supérieure de Télécommunications, Paris, France, in 2001 and Ecole Fédérale Polytechnique de Lausanne in Switzerland in 2002. His research interests in signal and image processing include wavelets and multiscale analysis, statistical modeling, and sensor networks.

Dr. Baraniuk received a NATO Postdoctoral Fellowship from NSERC in 1992, the National Young Investigator award from the National Science Foundation in 1994, a Young Investigator Award from the Office of Naval Research in 1995, the Rosenbaum Fellowship from the Isaac Newton Institute of Cambridge University in 1998, the C. Holmes MacDonald National Outstanding Teaching Award from Eta Kappa Nu in 1999, the Charles Duncan Junior Faculty Achievement Award from Rice University in 2000, the ECE Young Alumni Achievement Award from the University of Illinois in 2000, and the George R. Brown Award for Superior Teaching at Rice University in 2001 and 2003. 